LA-7469-MS

Informal Report

UC-71

Issued: September 1978

\title{
Cargo Response to Railcar Impact and Tiedown Load Analysis
}

\author{
Robert J. Bartholomew
}

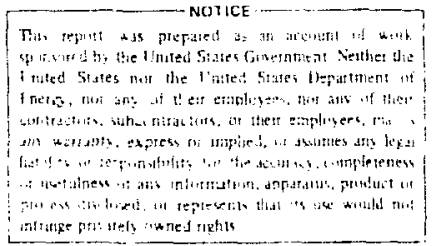


CARGO RESPONSE 'IO RAITCAR IMPACT AND 'TIEDOWN LOAD ANALYSIS

by

Robert J. Bartholomew

\begin{abstract}
An analytical study is described in which we investigated the loads produced during coupling of railcars carrying heav shipping containers. The structural model of the impact event is represented by a lumped-parameter technique. Each discrete ma'is lump possesses longitudinal, vertical, and rotational degrees of Ereedom. The resulting computer simulation provides Eor nonlinvar railcar coupler stiffness and linear damping forces in the coupler and container tiedowns. Results include response to paramelric variations in container weight, impact speed, and tiedown stiffness. Container dynamic response and tiedown loads are found to depend heavily on these parameters. Also, railcar bending and subsequent vertical motion are shown to be important contribitors to these responses. When experimentally substantiated, the nodel developed can serve as a useful tool in the design and evaluation of shipping container tiedown structures.
\end{abstract}

\title{
[ . INTRODUCTION
}

When massive energy materials containers are moved by rail, consideration must be given to forces produced by railcar couple: impacts. These forces may be large and can affect the container, its tiedown structure, and the railcar. Few rational dynamic analyses exist for the froblem of 
dynamic interaction between these three systems. Current design practice employs static analysis procedures using empirical load factors to design tiedown systems, but no dynamic analysis has confirmed the applicability of these static analysis methods. There is a need to develop a computer model that will handle the vertical, rotational, and longitudinal motions of both the container and the railcar. This is especially important for the heavier spent nuclear fuel containers that are transported by railcar.

Task IV of the FY 1977 work proposed by Los Alamos Scientific Laboratory (LASL) to the Division of Environmental Control Technology, Department of Energy, described an analytical study of the interaction of shipping containers, both with railcar and truck tiedowns. 'The analytical study of railcar impact during coupling was proposed Eor FY 1977 and is the subject of this report. The analysis of truck tiedowns is not included in this report, but the method developed here is generically the same. This report contains a summary of the railcar impact analytical study with sfecific conclusions, the general method of analysis, and a discussion of the analytical results. The accompanying appendices contain the basic data used to describe the analytical model, detailed equations and the digital computer mechanization of those equations, and a user's manual.

\section{SUMMARY AND CONCLUS IONS}

An analytical model of railcar impact during coupling was developed using a lumped structural parameter technique. ${ }^{2}$ The model is appropriate for horizontally laded, flexible containers and most flatbed railcars. A vertically laded rigid container can also be simulated. A digital computer simulation of this model, RICTL, standing for Railcar Impact Container Tiedown Loads, has been developed by LASL. This simulation, as prasently conceived, has provision for a nonlinear coupler as the only nonlinearity. However, the program is written so that nonlinear subroutines can be easily added to the tiedown configuration and to other longitudinal memburs of the railcar. With RICTL in its present form, we have studied the effects of variations in certain analytical model parameters. These parameters include container weight, impact speed, and tiedown stiffness (and corresponding damping). The effects 
of varying these parameters include the response accelerations of the railcar and the container and tiedown lnads. The results of these parametric studies indicate that the response accelerations and tiedown loads tend to increase with container weight, to vary approximately linearly with impact velocity, and to decrease with lower tiedown stiffness. The peak deceleration and coupler loads are relatively unaffected by these paramcter changes except for their approximately linear variation with impact velocity.

The major conclusions of this study are:

(1) Vertical plane dynamics and bending of both the railcar and the container are importani.

(2) Container accelerations and bending moments are low compared with those of the railcar. However, this was expected, because the coupler impact speeds were low. Impacts at higher speeds may result in considerably higher container loads.

(3) The results of our simulations are comparable to results ubtained using other analysis methods ${ }^{3,4}$ that are more 1 imited in application. For example, these other methods do not include vertical jynamics effects.

(4) The running time for the simulation on the computer is short, showing that economical analysis is possible.

(5) Before RICTL can he considered as an adequate design tool to evaluate tiedown loads, a degree of correlation should be established between r.ICTL and actual impact tests.

\section{METHOD OF ANALYSIS}

The structural dynamic method of analysis used to study the problem of coupling impact for a railcar carrying an energy material shipping container is based on the lumped parameter technique of structural modeling. Figure 1 shows a side-view schematic of a specific railcar ${ }^{4}$ and coupler, a shipping container with its tiedown configuration, and the mass lumping schematic with degrees of freedom assigned to each lumped mass. Mass 11 represents the initially stationary train that the railcar strikes. The container geometry is based on the NFS-M100 cask system, but we considered three weights--13.5, 27.2, 


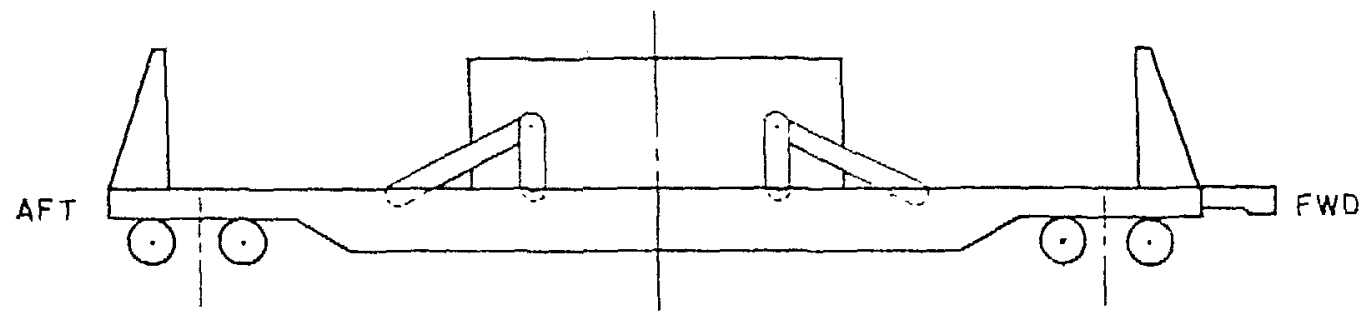

(9) (9) (19)
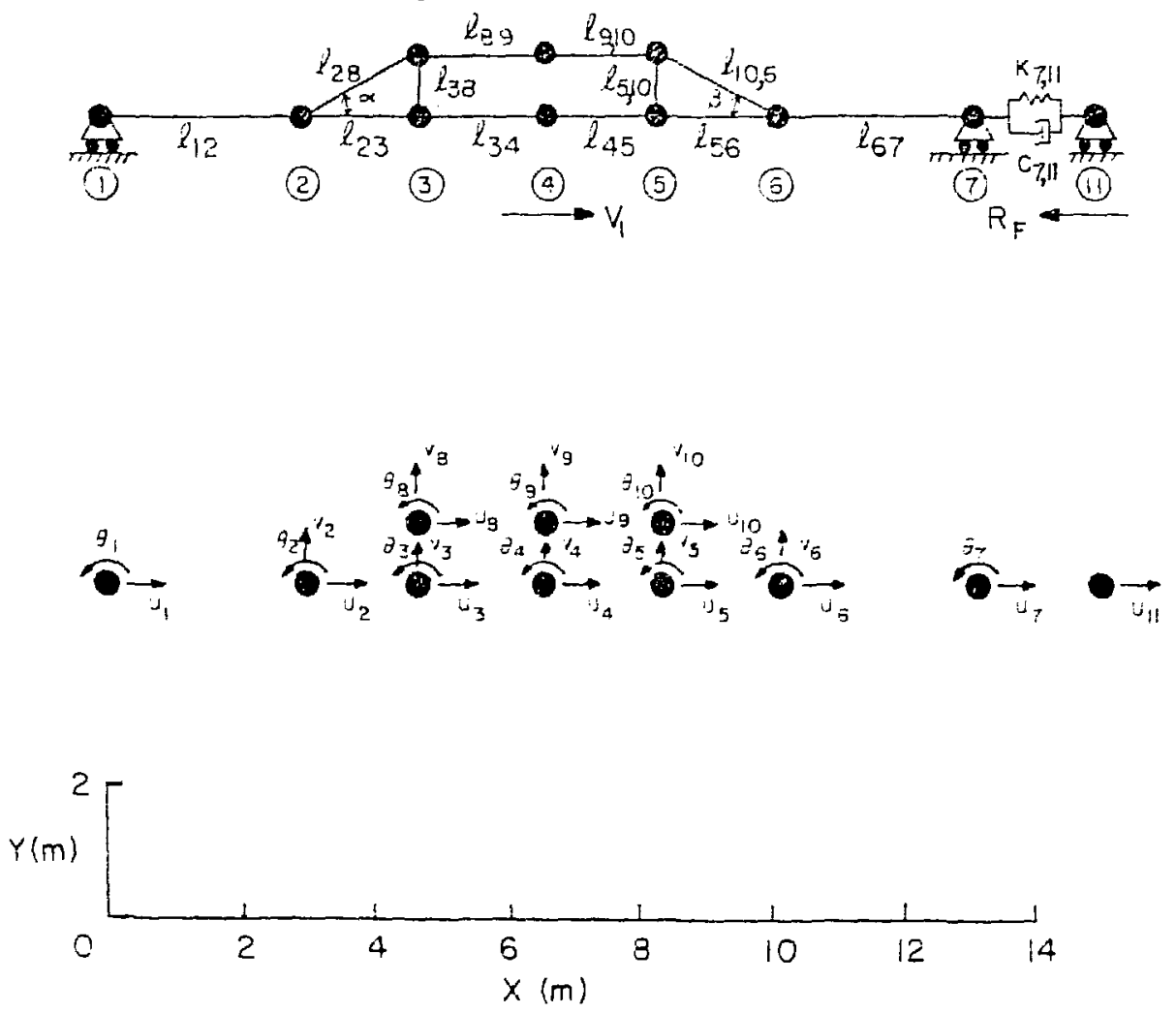

Eig. 1.

Structural modeling technique for railcar, container, and coupler. 
and 63.5 metric tonnes. The mass lumps 1-7 represent the moving railcar, and mass lumps 8-10 represent the shipping container. The mass lumps are interconnected by massless springs or dampers as indicated by the members designated $i_{i, j}$ between mass lumps $i$ and $j$, where $i, j=1,2 \ldots n$. The nonlinear coupler is designated by stiffness $k_{7,11}$ and damping $c_{7,11}$ as shown in Fig. 1. The impact velocity $\left(V_{I}\right)$ is given as the initial velocity in the direction shown for each of the ten masses composing the railcar-container structural dynamic system.

The lower schematic in Fig. I indicates the motion degrees of Ereedom for the system. The designation $u_{i}$ refers to the positive horizontal displacement of the $i^{\text {th }}$ mass; $v_{i}$ refers to the positive vertical displacement of the $i^{\text {th }}$ mass; and ${ }^{i}$ refers to the positive rotational motion of the $i^{\text {th }}$ mass. $V_{I}$ refers to the horizontal velocity with which the railcar carrying the shipping container strikes the initially stationary train. There are 29 degrees of freedom. The dynamic equations of motion in matrix form are:

$$
|M|: \ddot{x}+|C| \cdot \dot{x}+|K|: x \cdot=: F
$$

with initial conditions

$$
\dot{x}_{i}=\dot{u}_{i}=v_{I}(i=1,2,3 \ldots 10) \text { and }
$$

all other $\dot{\mathrm{x}}_{\mathrm{i}}=0$.

Also, in the eleventh equation of Eqs. (1), $\mathrm{F}_{11}=-\mathrm{R}_{\mathrm{F}}$, the sliding friction force of the initially stationary train being struck (Fig. 1). All other $: F ;=0$. The vector $x$ refers to the appropriate absolute motion of each of the 29 degrees of freedom; $|M|$ refers to the mass and mass-moment-ofinertia matrix; $|K|$ refers to the stiffness matrix; and $|\mathrm{C}|$ refers to the damping matrix. Equations (1) constitute a set of 29 second-order nonlinear differential equations, which are solved in the digital computer program RICTL.

The mass matrix $|M|$ is a diagonal matrix with 29 elements derived from railcar and container mass and mass-moment-of-inertia data. Appendix $A$ includes the numerical data and lumping methods used. The stiffness matrix [K] is derived in the standard way ${ }^{2}$ by first obtaining a transformation 
matrix ! f from absolute to relative coordinates. The axial and bending component stiffnesses $\mid$ are as given for beams, ${ }^{2}$ and the resulting stiffness matrix $|\mathrm{K}|$ was Eormulated by the matrix product

$$
|k|=10^{\mathrm{T}}|1 \cdot 1| \cdot \mid,
$$

where $1:{ }^{T} \mid$ is the transpose of $1: 1$.

The damping matrix $|C|$ was formulated by first assuming linear viscous damping in the tiedown members and in the coupler. These are the only damping forces included. They were formed in a relative damping matrix |il, and then $|C|$ was formulated by the matrix product

$$
|C|=1 \cdot{ }^{T}|1 \cdot| 1 \cdot 1 .
$$

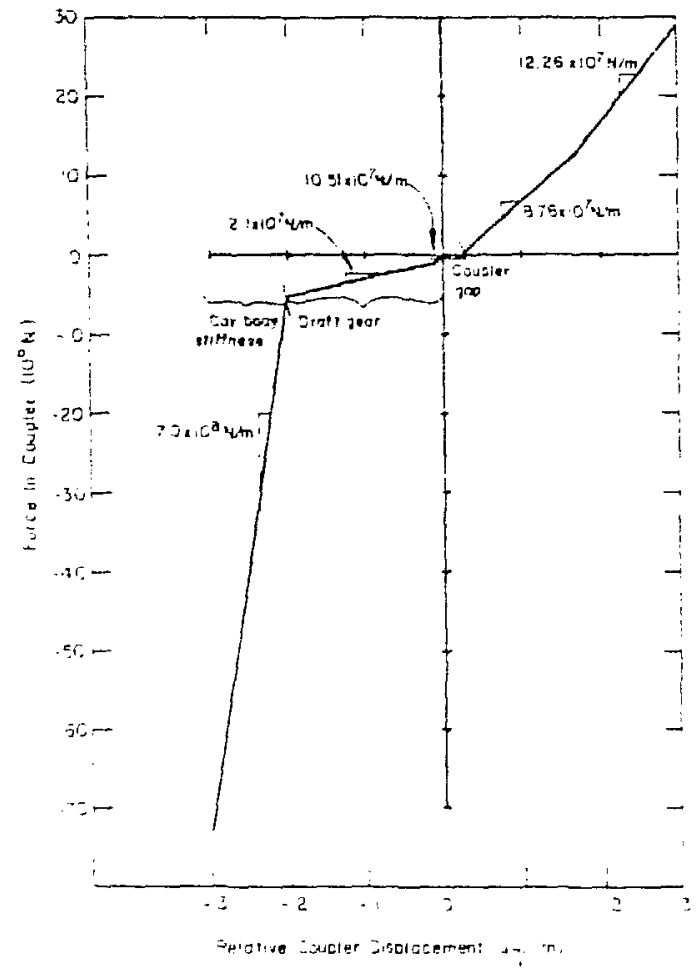

Fin. 2 .

anlinear stiffuces function for satuler.

The nonlinear spring $\left(k_{7,11}\right)$ associated with the draft gear of the coupler was assumed to be a standard Miner RF-333 draft gear. ${ }^{3}$ Figure 2 shows the force deflection characceristics of this coupler. A subroutine was written that updates the $k_{7,11}$ element in $|k|$ based on the current (time dependent) relative displacement in the coupler $\left(u_{7,11}=u_{11}-u_{7}\right)$.

A Runge-Kutta numerical integration routine was used to solve Eqs. (1). A constant time step increment was used, and the results of computer runs indicate that a sufficiently small time step for convergence does not require excessive computer time. This will be discussed later in the section on results.

The constraint forces (including tiedown loads) were computed from the following relationships for every time step:

$$
: x_{r e I}:=1: 1: x !
$$




$$
\begin{aligned}
\dot{\mathrm{X}}_{\text {rel }} & =1 \cdot \dot{\mathrm{x}} \cdot \\
\mathrm{F}: & =1 \cdot 1 \mathrm{x}_{\text {rel }}+|\because| \dot{\mathrm{x}}_{\text {rel }}:
\end{aligned}
$$

where F. the constraint force vector, and

$$
\begin{aligned}
& x_{r e l}, \dot{x}_{r e l} \text { the relative displacement and velocity of the } \\
& \text { fands of each member carrying a constraint force. }
\end{aligned}
$$

The detailed ratrices and equations of motion are given in Appendix $B$. The 1 isting of the computer program code RICTL is given in Appendix $C$. The RICTL user's manual is given in Appendix D.

IV. DISCUSSION OF RESULTS

The analytical results were obtained using the RICTL code with the model described in Appendix A. The study was parametric in nature with container weight, impact velocity, and tiedown stiffness and damping as parameters. The results of interest include peak values of the time profile of

(1) horizontal deceleration of the railcar at the point of impact,

(2) vertical response acceleration of the railcar at struck-end container attachment,

(3) horizontal deceleration of the container c.g.,

(4) vertical acceleration of the container c.g.,

(5) struck-end tiedown load horizontal component,

(6) itruck-end tiedown load vertical component,

(7) struck-end tiedown loar bending moment,

(8) coupler longitudinal force,

(9) ilaximum railcar bending moment, and

(10) maximum container bending moment.

In addition, typical time profiles of the above results for the heaviest container-railcar configuration striking an initially stationary train at the highest coupling velocity are included for the significant time portion of the impact event. The tiedown loads for the time profiles were expressed in terms of the total load of the two struck-end tiedowns (which produced the maximum 


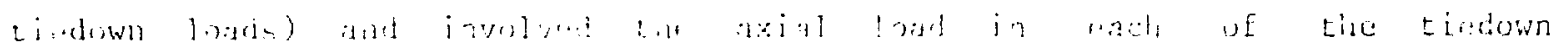
inembers "5, 10 and 70,6 . These axial loads can be converted to equivalent horizontal and vertical loats and a bending moment of a single nember atrached rigitly betweon Masis 10 and Mass 5. The relationships are:

$$
\begin{aligned}
& F_{\text {Horiz. }}=F_{10,0} \text { cis: } \therefore, \\
& F_{\text {Vert }}=F_{10,0} \sin \therefore+F_{10, j}, \\
& 3 M_{5}=5,10 F_{10,5} \cos \theta, \\
& \sin \therefore=\frac{5,10}{10,6},
\end{aligned}
$$

and

$$
\text { coss } \therefore=\frac{36}{111,6}
$$

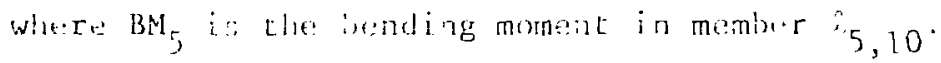

Figire 3 shows the variatiun in pak accaleration with the container. weight for tiu variablas of interest. The significant result shown in Fig. 3 is that, for heavy containers, the vertical acceleration of the railcar is nearly as large as the horizontal decelaration. This result imphasizes the relative importance of vertical dynamics compared with horizontal dynamics. This implies that bending effects of the container ale important in considering tiedown loads. Figure 4 shows the peak tiedown load variation with container weight. This plot confirms the fact that bending effects in the tiedowns need to be considrred. Although the net vertical component is small compared with horizontal tiedown loads, the bending moment is certainly significant.

Figure 5 shows the peak coupler longitudinal forces, maximum railcar bending moment, and maximum container bending moment, each expressed as a function of container weight. For the range of weights considered, we can see that the peak coupler load increases only slightly with weight and is about $5 \times 10^{6} \mathrm{~N}\left(1.124 \times 10^{6} 1 \mathrm{~b}\right)$. The maximum railcar bending moment follows a similar variation with weight and is largest for the heaviest container, 


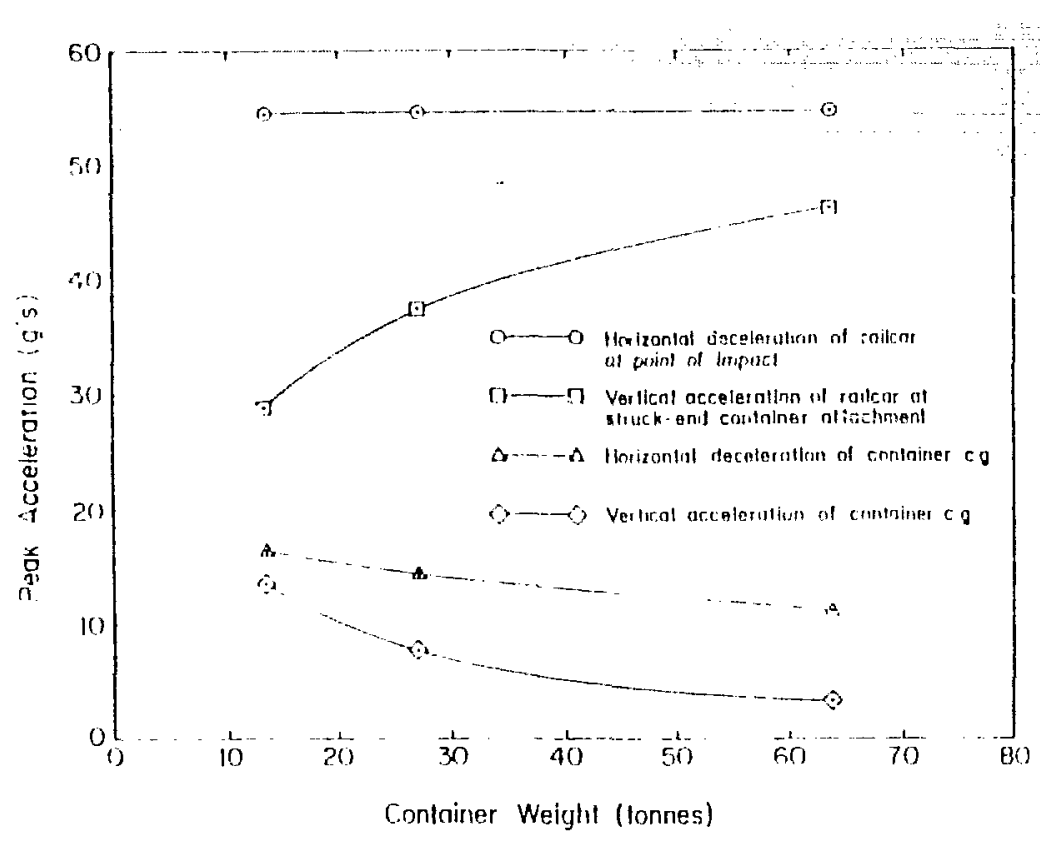

Fig. 3.

peak acceleration during impact at $17.7 \mathrm{~km} / \mathrm{h}(11 \mathrm{mph})$.

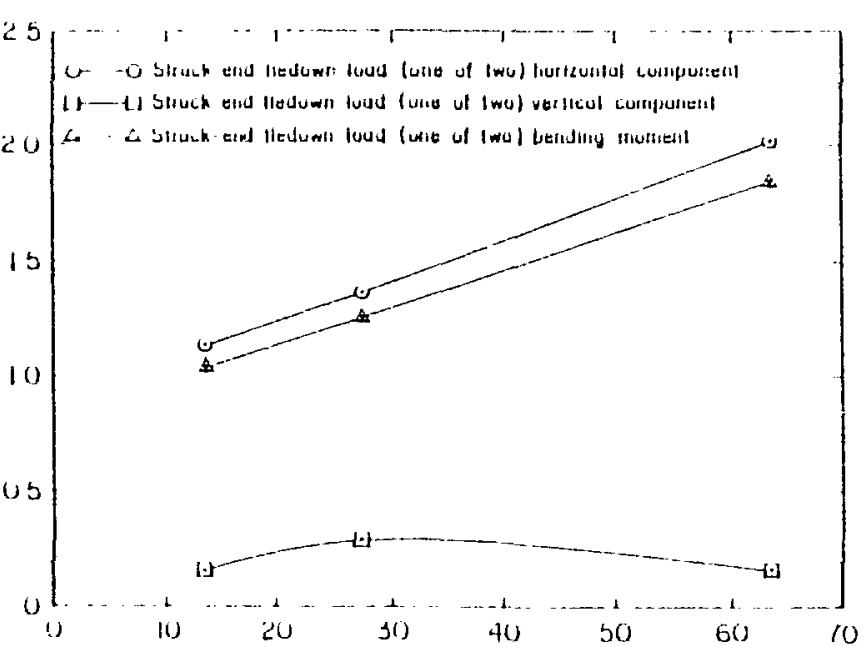

Fig. 4.

Peak tiedown loads during impact at $17.7 \mathrm{~km} / \mathrm{h}$ (11 $\mathrm{mph})$.

Corilaiser Weiglil (luilites)

Note: Weight of stationary train $=2.224 \times 10^{6} \mathrm{~N}(5000001 \mathrm{~b})$.

Resistance of stationary train $=4.448 \times 10^{5} \mathrm{~N}(1000001 \mathrm{~b})$. 
$1.75 \times 10^{6} \cdot \mathrm{n}-\mathrm{N}\left(15.49 \times 10^{6} \mathrm{in-1b}\right)$. The values of peak coupler load and railcar bending moment are comparable to values ilsed in the design of the railcar, namely, $4.448 \times 10^{6} \mathrm{~N}\left(1 \times 10^{6} \quad \mathrm{lb}\right)$ coupler 1 oad and $2.56 \times$ $10^{6} \mathrm{~m}-\mathrm{N}\left(22.5 \times 10^{6} \mathrm{in-1b}\right)$ bending monent, respectively.

Figures 6 through 8 show the variation of the same peak load variables as Figs. 3-5 with impact-speed as the parameter varied. The largest contalner weight, 63.5 tonnes, was used for these studies. The ourves all indicate that the peak loads vary linearly, or approximately linnarly, with impact speed. This is an antirely predictable result, since the inpulse is proportional to the momentum, which varies incarly with impact velocity. Variations from linearity are due to the peak loads being based on vibratory responses that peak at different relative times during the impact event. In the low-speed case $(8 \mathrm{~km} / \mathrm{h})$, the coupler load peaks at $0.006 \mathrm{~s}$ when the coupler stiffness is high $\left(10.51 \times 1.0^{7} \mathrm{~N} / \mathrm{m}\right)$. In all. of the higher speed cases, the coupler load peaks at $0.038 \mathrm{~s}$ when the coupler stiffness is low $\left(2.2 \times 10^{7} \mathrm{~N} / \mathrm{m}\right)$ during deflection of the draft gear. These times were obtained from a detailed computer printout of the results... In Fig. 8, the higher speed coupler loads are linearly related, but the low-speed coupler load is not on the same line. Thus, the nonlinear stiffness characteristic of the coupler accounts for the deviation from linearity of some of the peak response loads.

The peak horizontsi deceleration of the railcar is linearly related to impact velocity because it occurs 40 us after initial impact for all impact speeds. At this time the coupler stiffness is still in the tinear range, and the draft gear is undeflected. The container response acceleration peaks are ? inearly related to impact velocity because, al though they occur at times when the draft gear is deflecting, they occur at the same time $(0 . b i 2 s$ for horizontal response and $0.017 \mathrm{~s}$ for vertical response) for each impact velocity. Again, these times were obtained from a detailed computer printout of the results.

Figures 9 through 11 show the peak load variations of the 63.5-tonne container configuration with container tiedown axial stiffness. The tiedown stiffness embodies the same values of stiffness in each of the four members $\ell_{28}, \ell_{38}, \ell_{5,10}$, and $l_{10,6}$ Also, since the tiedown damping was assumed to vary with the tiedown stiffness, the damping constants were also varied by the same ratio as the stiffness. The most notable result is that response variables that depend on the tiedown interconnections to transmit 

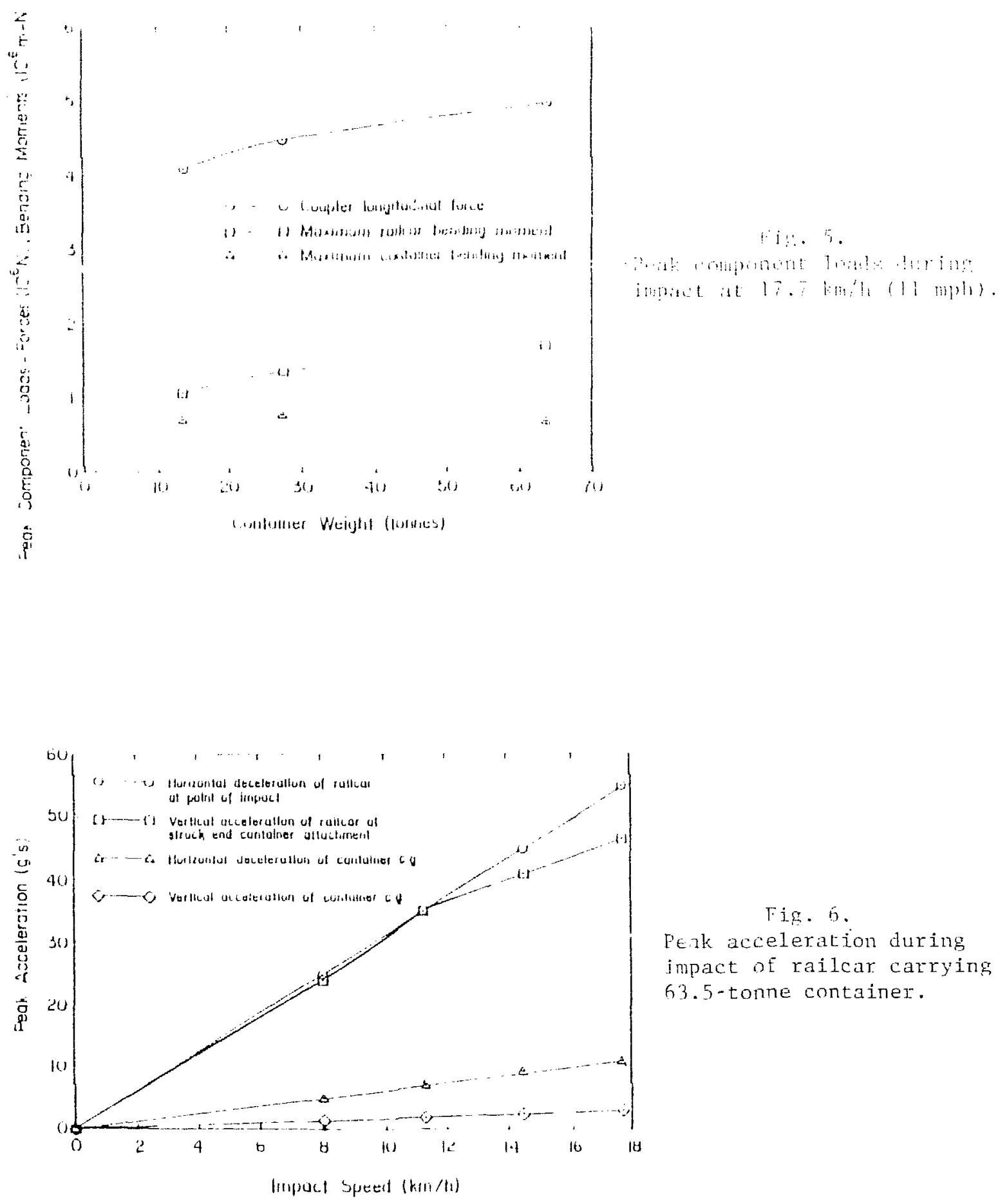

Note: Weight of stationary train $=2.224 \times 10^{6} \mathrm{~N}(500000 \mathrm{lb})$.

Resistance of stationary train $=4.449 \times 10^{5} \mathrm{~N}(1000001 \mathrm{~b})$. 


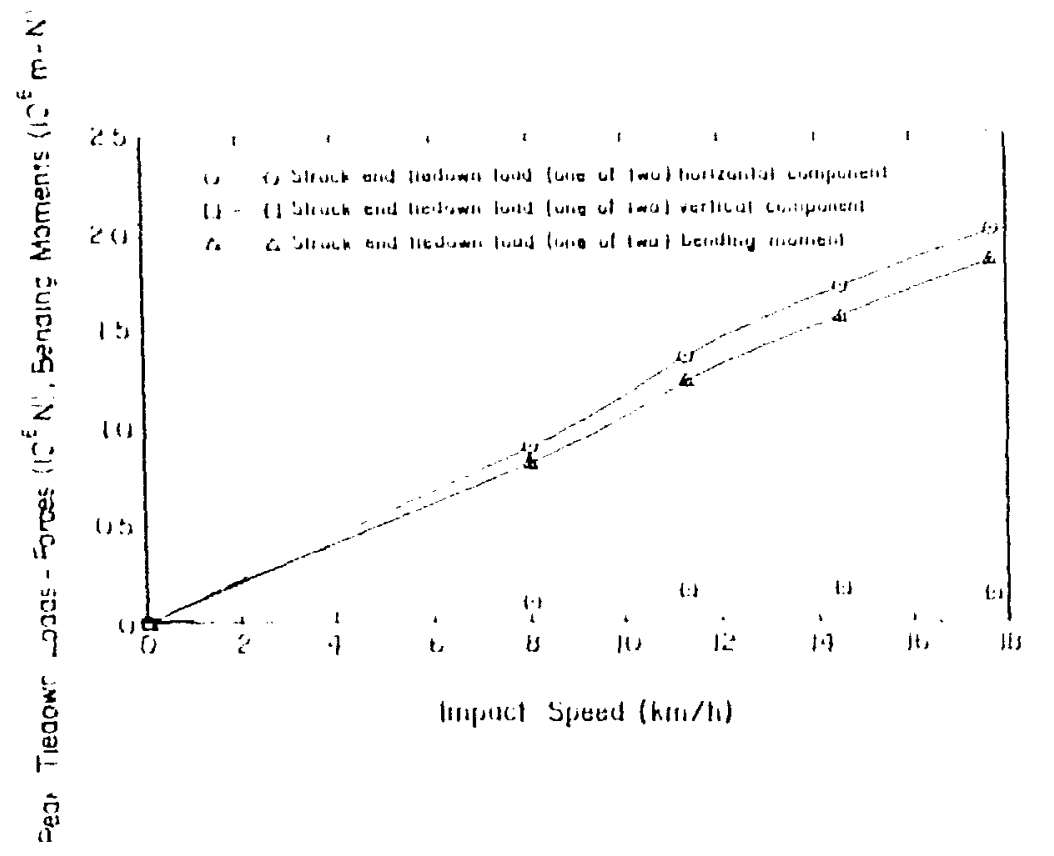

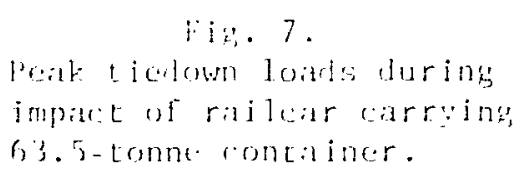

Fig. 8.

Peak component loads during impact of railcar carrying 63.5-tonne container.

Note: Weight of stationary train $=2.224 \times 10^{6} \mathrm{~N}(5000001 \mathrm{~b})$. Resistance of stationary train $=4.448 \times 105 \mathrm{~N}(100000 \mathrm{lb})$. 


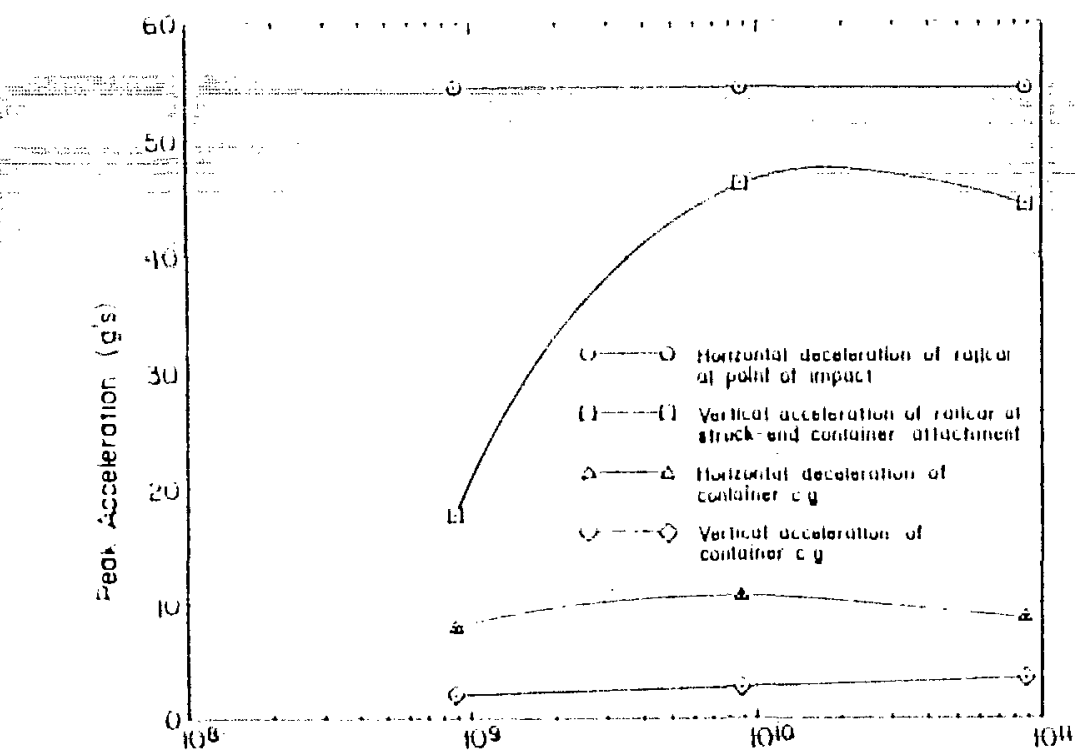

Liodown Axiul Stiffries\$ (Euch, Merrlot) (W/AI)

要

Note: Weight of stationary train $=2.224 \times 10^{6} \mathrm{~N}(50 \mathrm{r} 000 \mathrm{lb})$. Resistance of stationary train $=4.448 \times 10^{5} \mathrm{~N}(100000 \mathrm{lb})$.

Viedown Axial Stiffness (Each Mernber) (N/m)

范
() $100^{4}$

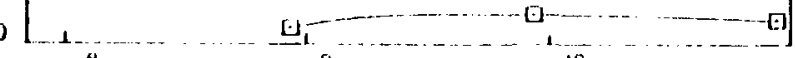

$10^{9}$

$$
10^{10}
$$

Fig. 9 .

Peak acceleration during impact at $1.7 .7 \mathrm{~km} / \mathrm{h}$ (11 mph) of railear carrying 63.5conne container.
Fig. 10 .

Peak tiedown loads during impact at $17.7 \mathrm{~km} / \mathrm{h}$ (11 mph) of railcar carrying 63.5tonne container. 
Loads are markedly reduced when the tiedown stiffness is reduced. The railcar vertical acceleration plot of Fig. 9 and the tiedown load plots ol Fig. 10 show this effect. The compler load and ralcar impact point deceleralion were unaffected by tidown stiffress variation.

Figures 12 through 1.4 show typical time profiles of the heaviest weight container-railcar configuration strikjng an initially stationary train at the highest coupling impact velocity $(17.7 \mathrm{~km} / \mathrm{h}$ or $11 \mathrm{mph})$. These plots are included to show the oseillatory behavior of the impact event. The impact deceleration has an apparent dominant frequency of $1.25 \mathrm{~Hz}$, which is revealed initially in the tiedown load of nember ' $(0,6)$ the tiedown having the dominant loading. This result appears to bo approximately in accord with the result abtained by Magnuson and Wilson in their mortel that has only longitudinal degrees of freedom for the ATM car tiedown structure (dominant frequency of $100 \mathrm{~Hz}) .^{3}$ The magnitude of peak deceleration that we obtained ( $54.5 \mathrm{~g}^{\prime} \mathrm{s}$ ) also compares with the Magnuson and Wilson results For cargo weights between $178000 \mathrm{~N}$ and $445000 \mathrm{~N}$, which gave $52 \mathrm{~g} \mathrm{~g}^{\prime} \mathrm{s}$, and $58 \mathrm{~g}^{\prime} \mathrm{s}$, respectively, for a spent fuel cask system with $3.2 \mathrm{~mm}$ free travel space. The configuration of Magnuson and wilson and our configuration are not exactly the same, so a direct comparison is not possible. However, the closeness of the two results for longitudinal motion variables is of interest. For 50 ms of elapsed time (500 time steps), the CDC 7600 CPU time was slight1y over two minutes so that the simulation is economical to use.

In conclusion, the results indicate that the RICTL simulation of railcar coupling impact dynamics produces results that are plausible when compared with resilts of other impact anslyses. 3,4 The analysis method applied to the design of cargo tiedowns should be a significant improvement over current static design methods ${ }^{5}$ that employ equivalent empirically derived load factors in the design of tiedowns. Also, vertical motion and bending are found to be important effects to be considered in the design of tiedown structure. More cannot be said about the adequacy of the simulation until a degree of correlation is established between the RICTL model and actual impact tests. 
E

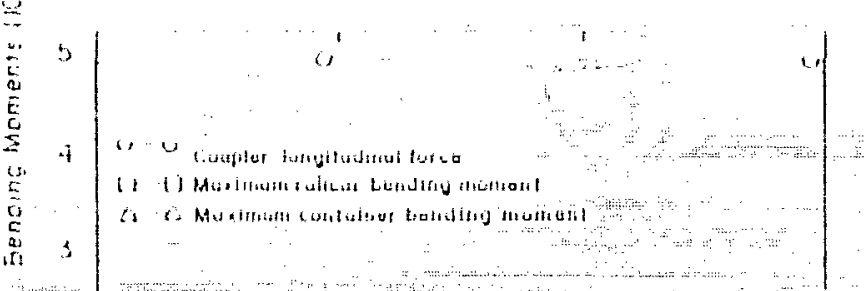

$\operatorname{sen}_{4}-\cos _{2}$

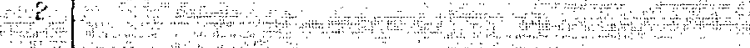

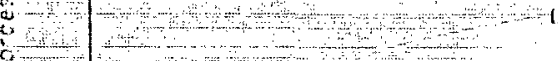

$r_{1}=-10$

$\begin{array}{lll}1 & & \\ 0 & & 0\end{array}$

50

$=1$

$11 i^{i}$

$100^{11.2}$

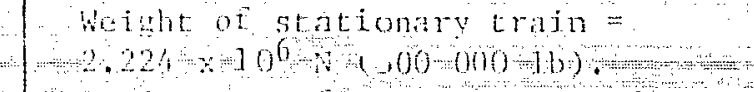

Fis. li.

Port componont lomis during impact at $17.7 \mathrm{~km} / \mathrm{h}$ (1) mph of railcar adrying 63.5-tomo container.

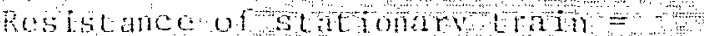
$114.448 \times 162+(10000010)$

a. $\quad$ a

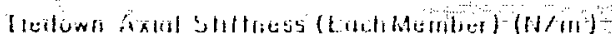

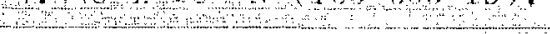

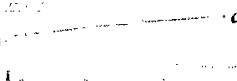

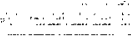

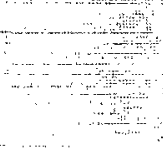

(1).....

a. $\ldots \ldots$

3

Fig. 12 .

Acceleration time profiles for railcar carrying 63.5 -tonne container striking 226.8-tonne stationary train at $17.7 \mathrm{~km} / \mathrm{h}$ (11 mph). Resistance of stationary train $=4.448 \times 10^{5} \mathrm{~N}(10000013)$.

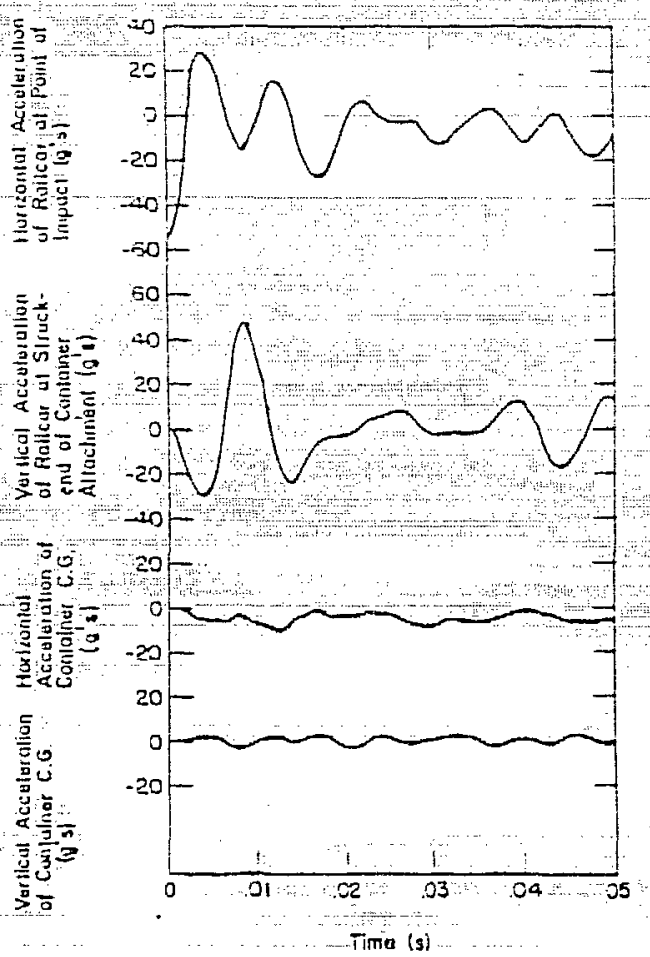




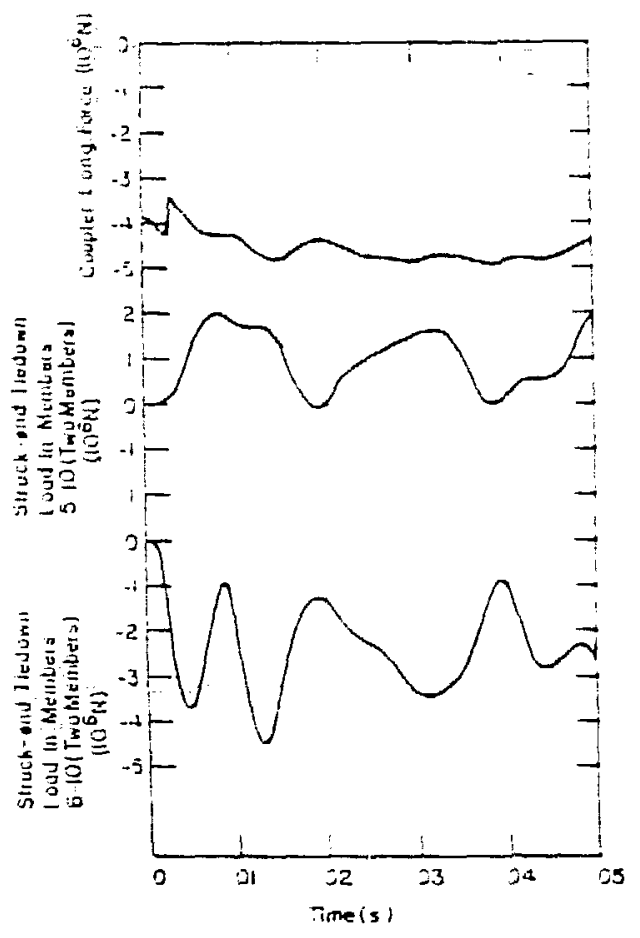

Fig. 13.

Coupler and tiedown load time profiles for railcar carrying 63.5-tonne container striking 226.8-tonne stationary trajn at $17.7 \mathrm{~km} / \mathrm{h}(11 \mathrm{mph})$.

Fig. 14.

Bending moment time profiles for railcar carrying 63.5-tonne container striking 226.8-tonne stationary train at $17.7 \mathrm{~m} / \mathrm{h}$ (11 mph).

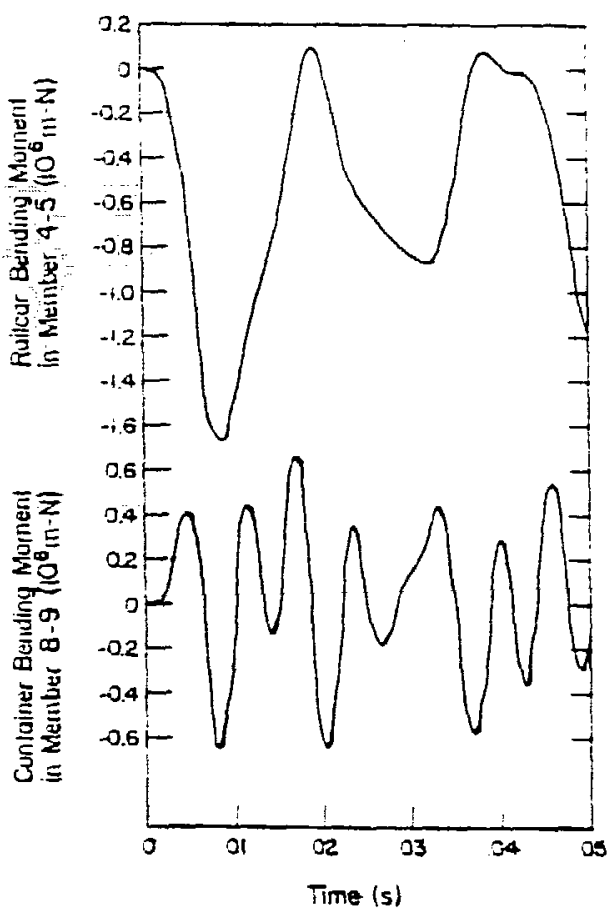

Note: Resistance of stationary train $=4.448 \times 10^{5} \mathrm{~N}(1000001 \mathrm{~b})$. 
The efforts of T. A. Butler,.E. G. Endebrock, J. B. Payne, W. F. Corcoran, and J. W. Straight in helping to code and debug the RICTL program are gratefully acknowledged.

\section{REFERENCES}

1. Los Alamos Scientific Laboratory (LASL), Schedule 189c No. A482, "Development of Analytical Methods for Accident Damage Analysis of Type B Packages," Task No. LS-82-1-7-B to Division of Environmental Control Technology, ERDA (May 3, 1976).

2. Walter C. Hurty and Moshe F. Rubinstein, Dynamics of Structures (Prentice-Hal1 Inc., New Jersey, 1964), pp. 38-47,285.

3. Clifford F. Magnuson and Leonidas T. Wilson, "Shock and Vibration Environments for Large Shipping Containers on Railcars and Trucks," Sandia Laboratories Albuquerque report SAND 76-0427 NUREG 766510 (June i 977.).

4. Association of American Railroads, "Specifications for Design Fabrication and Construction of Freight Cars Adopted Sept. 1, 1964," Appendix L, "Stress Analysis for 50 ton 53 ft. 6 in. Bulkhead Flat Car," Chicago, I11. (January 1966).

5. US Energy Research and Development Administration, Division of Reactor Research and Development, "RDT Standard-Fuel Shipping Container Tiedown for Rail Transport," DRAFT report RDT F8-12 (March 1977). 
APPENDTX A

MALTTLCAL NODEL DATA

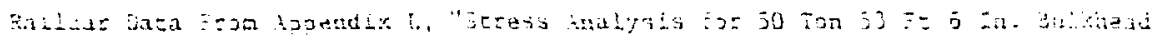

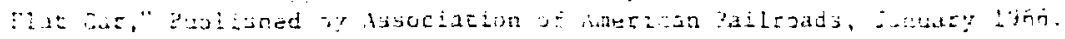

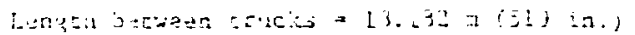

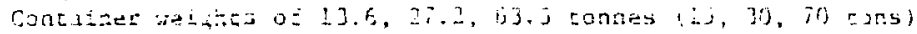

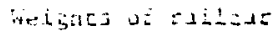

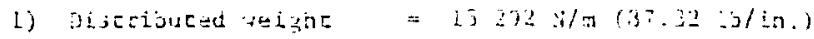

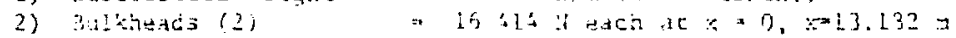

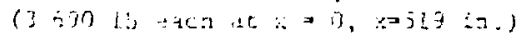

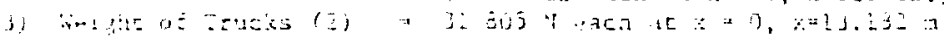

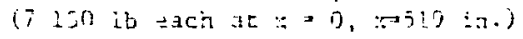

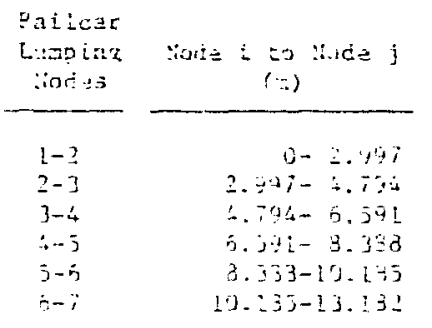

\begin{tabular}{|c|c|c|}
\hline $\begin{array}{l}\text { Uezoer } \\
\text { Lenthth } \\
3(n)\end{array}$ & $\begin{array}{c}\text { Adight } \\
\text { Distriburion } \\
\text { (:i) }\end{array}$ & $\begin{array}{c}\text { Yetrier Sectional } \\
\text { Areas } \\
r^{2}\end{array}$ \\
\hline 2. 347 & $\therefore 334$ & (j. $9: \therefore 73$ \\
\hline 1.907 & $27 \& 11$ & $9.95 \mathrm{si}$ \\
\hline 1.7? & $27 \div$ & 0.0535 \\
\hline $1 . \therefore 17$ & 27 i & $0.053:$ \\
\hline 1. .7. & $27 \leq 52$ & $2.25 \%$ \\
\hline 2.997 & 45834 & $0.0: 3: 3$ \\
\hline
\end{tabular}

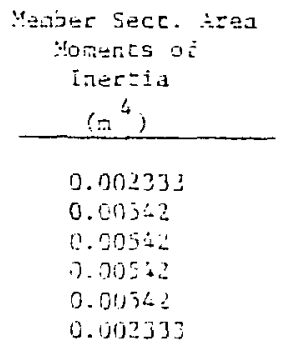

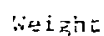

ieight bijtzibution ?:I)

13.5 zonne 2 i. i torne

and $;$

$3 A-8 F=$

$9 A-9=$

$10 \therefore-10 \bar{Y}$

$$
\begin{aligned}
& 3.3-5.7 \\
& 5.7-3.3 \\
& 7.5-9.3
\end{aligned}
$$

1.3

1.8

1.8

$\begin{array}{ll}44 & 482 \\ 4 \therefore \quad 482\end{array}$

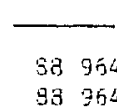

44482

88964

Menier Sectional

$$
\text { ireas }\left(a^{2}\right)
$$

$\begin{array}{rcc}3 A-3 E & \frac{(3)}{2(\mathrm{~m})} \\ 93-97 & 3.5-5.7 & 1.8 \\ 10.1-197 & 5.7-7.5 & 1.3 \\ & 7.5-3.3 & 1.3\end{array}$

\section{3. ó tonnt.}

0.09543
0.09548
0.09548

\section{2 tona.}

0.13805

0.13305

0.13800

\begin{tabular}{|c|c|c|c|c|}
\hline$(7)$ & $2(\pi)$ & 13.5 tonne & 27.2 t.2r:e & 63.5 zorae \\
\hline .7 & 1.3 & 0.007679 & $0.0231 ?$ & 0.11222 \\
\hline .7 & 1.8 & 0.007679 & 0.02312 & 0.11222 \\
\hline $.5-$ & 1.3 & 0.007679 & 0.0212 & 0.12232 \\
\hline
\end{tabular}

$\frac{63.5=5 a n e}{0.23387}$
0.23387
0.23387

Yemier Sectional

$$
\text { Yonencs of Inertia (a }{ }^{4} \text { ) }
$$

$c_{i 2}-9=$

\begin{tabular}{|c|c|c|}
\hline 53.3 & $\cos n=$ & 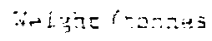 \\
\hline 207 & 535 & 13.6 \\
\hline 207 & 335 & 27.2 \\
\hline 207 & 535 & 63.3 \\
\hline
\end{tabular}

$10 \mathrm{i}-10 \mathrm{E}$

I. 3

0.007579

0.0223

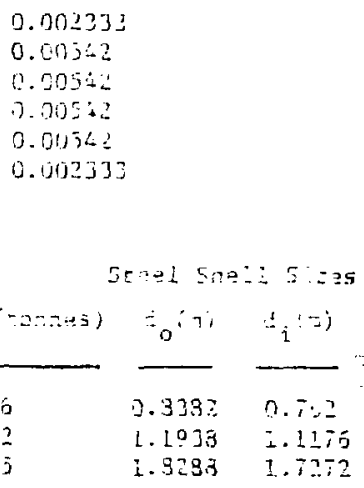

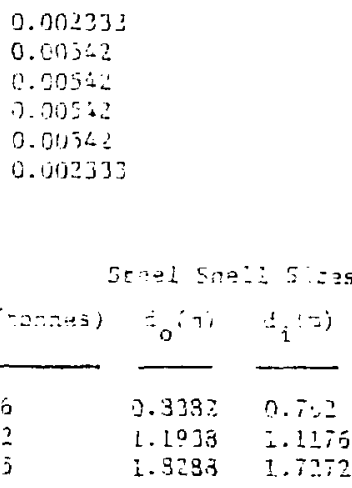

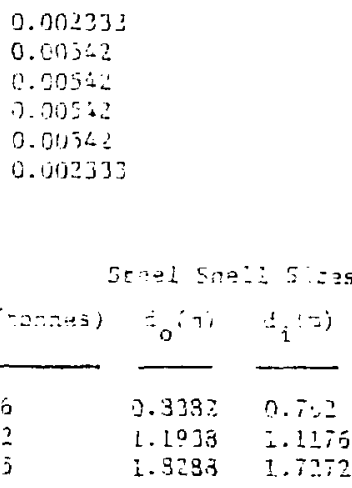

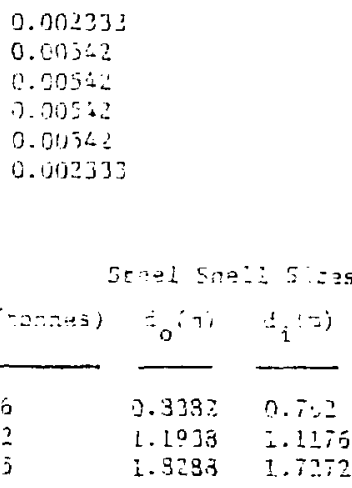

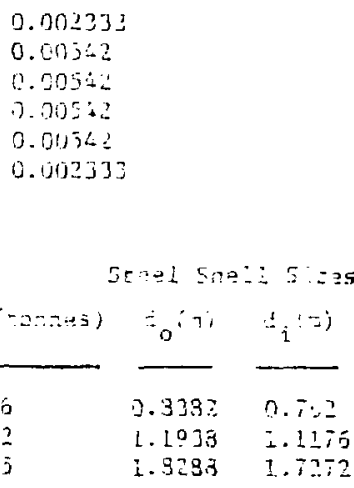


Lumped weights are obtained by

$$
\begin{aligned}
& W_{1}=\frac{W \text { Dist. } 1-2}{2}+16414+31805=71,136 \mathrm{~N}=\mathrm{W}_{7} \\
& \mathrm{~W}_{2}=\frac{\mathrm{W} \text { Dist. } 1-2}{2}+\frac{\mathrm{W} \text { Dist. } 2-3}{2}=36658 \mathrm{~N}=\mathrm{W}_{6} \\
& \mathrm{~W}_{3}=\frac{\mathrm{W} \text { Dist. } 2-3}{2}+\frac{\mathrm{W} \text { Dist. } 3-4}{2}=27481 \mathrm{~N}=\mathrm{W}_{4}=\mathrm{W}_{5} \\
& \mathrm{~W}_{8}=\mathrm{W}_{9}=\mathrm{W}_{10}=\mathrm{W} \text { Dist. } 8 \mathrm{~A}-8 \mathrm{~F}
\end{aligned}
$$

Weight Moments of Inertia are obtained by:

$$
\begin{aligned}
& \text { Railcar }-I_{i}=\frac{w_{i} d_{i}^{2}}{12}, d_{i}=0.7184 \mathrm{~m}(28.285 \mathrm{in.}), i=1,2,3,4,5,6,7 \\
& \text { Container }-I_{i}=\frac{w_{i} d_{i}^{2}}{16}, d_{i}=1.8288 \mathrm{~m} \text { (72 in.), } i=8,9,10
\end{aligned}
$$

\begin{tabular}{|c|c|c|c|c|c|}
\hline Lump (i) & $\mathrm{x}_{\mathrm{i}}(\mathrm{m})$ & $Y_{i}(\mathrm{~m})$ & $\mathrm{W}_{i}(\mathrm{~N})$ & $\mathrm{W}_{i}(\mathrm{~N})$ & $\mathrm{W}_{i}(\mathrm{~N})$ \\
\hline 8 & 4.794 & 0.9144 & $44 \quad 482$ & 88964 & 207585 \\
\hline 9 & 6.591 & 0.9144 & $44 \quad 482$ & 88964 & 207585 \\
\hline 10 & 8.388 & 0.9144 & $44 \quad 482$ & 88964 & 207585 \\
\hline
\end{tabular}

Lumped Data

$$
\begin{aligned}
& \text { Railcar }
\end{aligned}
$$

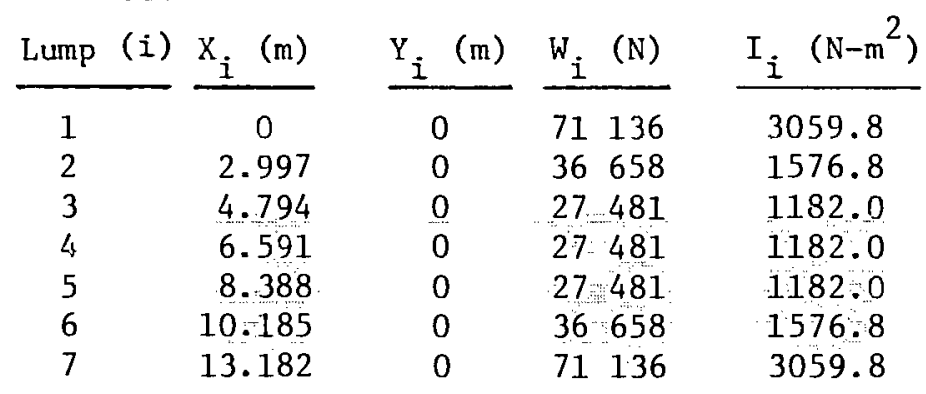

Container Weight $\quad 13.6$ tonne 27.2 tonne 63.5 tonne

Container Mass Moment of Inertia

13.6 tonne 27.2 tonne 63.5 tonne

$\begin{array}{ccccccc}\text { Lump }(\mathrm{i}) & \frac{X_{i}(\mathrm{~m})}{8} & \frac{Y_{i}(\mathrm{~m})}{4.794} & \frac{I_{i}\left(N-\mathrm{m}^{2}\right)}{0.9144} & \frac{I_{i}\left(N-m^{2}\right)}{9.298} & \frac{I_{i}\left(N-m^{2}\right)}{18.596} & \frac{43392}{433} \\ 9 & 6.591 & 0.9144 & 9298 & 18596 & 43392 \\ 10 & 8.388 & 0.9144 & 9298 & 18596 & 43392\end{array}$


Andul i (l'at)

$$
\begin{aligned}
& \text { Raticar } E_{R}=2.0684 \times 10^{31}(30000000 \text { pisi) } \\
& \text { Container } E_{C}=2.0684 \times 10^{11}(30000000 \text { psi) }
\end{aligned}
$$

Acceleration of gravity $\left(\mathrm{m} / \mathrm{s}^{2}\right)$

$$
r=9.80665\left(32.17 \mathrm{rt} / \mathrm{s}^{2}\right)
$$

\begin{tabular}{|c|c|c|c|}
\hline lember & 1). 1 Nominal & Nominal. & 10 Nominal. \\
\hline $2-$ & $1.226 \times 10^{5}$ & $1.226 \times 10^{6}$ & $1.226 \times 1$ \\
\hline $3-8$ & $1.226 \times 10_{5}^{5}$ & J. $226 \% 10^{6}$ & $1.226 \times 10^{7}$ \\
\hline $10-5$ & $1.226 \times 10_{5}^{5}$ & $1.226 \times 10^{6}$ & $1.226 \times 10_{7}^{7}$ \\
\hline $10-6$ & $1.226 \times 10^{5}$ & $1.226 \times 10^{6}$ & $1.226 \times 10^{\prime}$ \\
\hline
\end{tabular}

Tiedown Damping (Est. $\tau=0.05)-\left(\frac{\mathrm{N}}{\mathrm{m} / \mathrm{s}}\right)$

Tiedown Stiffness $(\mathrm{N} / \mathrm{m})$

\begin{tabular}{rllll} 
Member & 0.1 Nominal & & Nominal & $\frac{10 \text { Nominal }}{2-8}$ \\
\hline $2-756 \times 10_{8}^{8}$ & $8.756 \times 10^{9}$ & $8.756 \times 10^{10}$ \\
$3-8$ & $8.756 \times 10^{8}$ & $8.756 \times 10^{9}$ & $8.756 \times 10^{10}$ \\
$10-5$ & $8.756 \times 10_{8}^{8}$ & $8.756 \times 10^{9}$ & $8.756 \times 10_{10}^{10}$ \\
$10-6$ & $8.756 \times 10^{8}$ & $8.756 \times 10^{9}$ & $8.756 \times 10^{10}$
\end{tabular}

Impact Velocity $(\mathrm{km} / \mathrm{h}) \mathrm{V}_{T}=8,11.3,14.5,17.7$

Stationary Train - Weight $=226.8$ tonnes $-2.224 \times 10^{6} \mathrm{~N}(5000001 \mathrm{~b})$

- Resistance Force (coeff. of Friction of 0.2 ) = $4.448 \times 10^{5} \mathrm{~N}(100000 \mathrm{lb})$ 
The equations of motion for the railcar coupling impact dynamics problem are, as stated in the methods section of the report,

$$
[M]\{\ddot{x}\}+[C]\{\dot{x}\}+[K]\{x\}=\{F\}
$$

For che degrees of freedom indicated in Fig. 1 of the report we have the currespondence:

$$
\begin{aligned}
x_{1}=u_{1} & x_{16}=v_{6} \\
x_{2}=u_{2} & x_{17}=v_{8} \\
x_{3}=u_{3} & x_{18}=v_{9} \\
x_{4}=u_{4} & x_{19}=v_{10} \\
x_{5}=u_{5} & x_{20}=\theta_{1} \\
x_{6}=u_{6} & x_{21}=\theta_{2} \\
x_{7}=u_{7} & x_{22}=\theta_{3} \\
x_{8}=u_{8} & x_{23}=\theta_{4} \\
x_{9}=u_{9} & x_{24}=\theta_{5} \\
x_{10}=u_{10} & x_{25}=\theta_{6} \\
x_{11}=u_{11} & x_{26}=\theta_{7} \\
x_{12}=v_{2} & x_{27}=\theta_{8} \\
x_{13}=v_{3} & x_{28}=\theta_{9} \\
x_{14}=v_{4} & x_{5}
\end{aligned}
$$

The masses are:

$$
\begin{aligned}
& M_{11}=M_{1} \\
& M_{22}=M_{2}
\end{aligned}
$$

$$
\begin{aligned}
& M_{16,16}=M_{6} \\
& M_{17,17}=M_{8}
\end{aligned}
$$




\section{$M_{33}=M_{3}$}

$\mathrm{M}_{18,18}=\mathrm{M}_{9}$

$M_{44}=M_{4}$

$19,19=M_{10}$

$\overline{M_{55}}=M_{5}$

$M_{20,20}=I_{1}$

$M_{66}=M_{6}$

$M=M$

$M^{2}$

$M_{88}=M_{8}$

$\mathrm{M}_{99}=\mathrm{M}_{9}$

$M_{21,21}=I_{2}$ is

$M_{22,22}=I_{3}$

$\mathrm{M}_{23}, 23=-\mathrm{I}$

$M_{24}, 24=I_{5}$

$M_{10,10}=M_{10}$

$M_{25}, 25=\mathrm{I}$

$M_{11,11}=M_{11}$

$M_{26,26}=I_{7}$

$=M_{12,12}=M_{2}$

$\mathrm{M}_{27,27}=\mathrm{I}_{8}$

$\mathrm{M}_{13,13}=\mathrm{M}_{3}$

$M_{28,28}=I_{9}$

$M_{14,14}=M_{4}$

$M_{15,15}=M_{5}$

$M_{29,29}=110$

All other $M_{i j}=0$.

The damping matrix:

$$
\begin{aligned}
& r_{77}=c_{28} \\
& r_{88}=c_{38} \\
& r_{11,1}=c_{10,5}
\end{aligned}
$$

$$
\begin{aligned}
& Y_{12,12}=c_{10,6} \\
& Y_{29,29}=c_{7,11}
\end{aligned}
$$

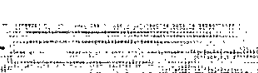

A1 other $\gamma_{i j}=0$.

The relative coordinates in terms of the absolute coordinates are given by the relations:

$$
\begin{aligned}
& u_{12}=u_{2}-u_{1} \\
& u_{23}=u_{3}-u_{2} \\
& u_{34}=u_{4}-u_{3} \\
& u_{45}=u_{5}-u_{4} \\
& u_{56}=u_{6}-u_{5}
\end{aligned}
$$

$$
\begin{aligned}
& u_{7,11}=u_{11}-u_{7} \\
& u_{89}=u_{9}-u_{8} \\
& u_{9,10}=u_{10}-u_{9} \\
& u_{28}=\left(u_{8} \cos \alpha+v_{8} \sin \alpha\right)- \\
& \quad\left(u_{2} \cos \alpha+v_{2} \sin \alpha\right) \\
& u_{38}=v_{8}-v_{3}
\end{aligned}
$$




$$
\begin{aligned}
& u_{67}=u_{7}-u_{6}-u_{10,5}=v_{10}-v_{5} \\
& u_{10,6}=\left(u_{6} \cos \beta-v_{6} \sin \beta\right)=\left(u_{10} \cos \beta-v_{10} \sin \beta\right)
\end{aligned}
$$

where

$$
\cos \alpha=\frac{\ell_{23}}{\ell_{28}}, \sin \alpha=\frac{\ell_{3}}{\ell_{28}}, \cos \beta=\frac{\ell_{56}}{\ell_{10,6}}, \sin \beta=\frac{\ell_{1}, 10}{10,6}
$$

$$
\begin{array}{lr}
v_{12}=v_{2}-l_{12} \theta_{1} & \theta_{12}=\theta_{2}-\theta_{1} \\
v_{23}=v_{3}-\left(v_{2}+l_{23} \theta_{2}\right) & \theta_{23}=\theta_{3}-\theta_{2} \\
v_{34}=v_{4}-\left(v_{3}+l_{34} \theta_{3}\right) & \theta_{34}=\theta_{4}-\theta_{3} \\
v_{45}=v_{5}-\left(v_{4}+l_{45} \theta_{4}\right) & \theta_{45}=\theta_{5}-\theta_{4} \\
v_{56}=v_{6}-\left(v_{5}+l_{56} \theta_{5}\right) & \theta_{56}=\theta_{6}-\theta_{5} \\
v_{67}=-\left(v_{6}+l_{67} \theta_{6}\right) & \theta_{67}=\theta_{7}-\theta_{6} \\
v_{89}=v_{9}-\left(v_{8}+l_{89} \theta_{8}\right) & \theta_{89}=\theta_{9}-\theta_{8} \\
v_{9,10}=v_{10}-\left(v_{9}+l_{9,10} \theta_{9}\right) & \theta_{9,10}=\theta_{10}-\theta_{9}
\end{array}
$$

The $[\beta]$ matrix is set up as follows from the relative coordinatel absolute coordinate equations.
1. All $\beta_{i j}=0$ except for those that follow $\left(\beta_{i j} \equiv \operatorname{BETA}(i, j)\right)$
2. $\operatorname{BETA}(1,1)=-1.0$
12. $\operatorname{BETA}(6,6)=-1.0$
$3 . \quad \operatorname{BETA}(1,2)=1.0$
13. BETA $(6,7)=1.0$
4. $\operatorname{BETA}(2,2)=-1.0$
14. $\quad \operatorname{BETA}(7,2)=-\ell_{23} /{ }^{\bar{x}}{ }_{28}$
5. BETA $(2,3)=1.0$
15. $\operatorname{BETA}(7,8)=l_{23} /{ }_{28}$
6. $\operatorname{BETA}(3,3)=-1.0$
16. BETA $(7,12)=-l_{38} /{ }_{28}$
7. $\operatorname{BETA}(3 ; 4)=1.0$
17. $\quad$ BETA $(7,17)=l_{38} /^{l}{ }_{28}$
8. BETA $(4,4)=-1.0$
18. $\operatorname{BETA}(8,13)=-1.0$
9. $\quad \operatorname{BETA}(4,5)=1.0$
19. $\quad \operatorname{BETA}(8,17)=1.0$
10. $\operatorname{BETA}(5,5)=-1.0$
20. $\operatorname{BETA}(9,8)=-1.0$
11. $\operatorname{BETA}(5,6)=1.0$
$21 . \operatorname{BETA}(9,9)=1.0$ 
22. BETA $(1.0,9)=-1.0$

23. $\operatorname{BETA}(10,10)=1.0$

24. BETA $(11,15)=-1.0$

25. ВЕГА $(11,19)=1.0$

26. BELA $(12,6)=\frac{6}{56 / 1} 10,6$

27. $\therefore$ ВеГА $(12,10)=-9,10,6$

$28=B E T A(12,16)=-1,10,1 / 10,6$

$29+\mathrm{BETA}-12 ; 19)=2,5,10^{/ 2} / 10,6$

$30=\mathrm{BETA}(13,12)=1.0$

31: $\quad \operatorname{BETA}(13,20)=2=2$

32. BETA $(14,12)=-1.0$

33. BETA $(14,13)=1.0$

34. BETA $(14,21)=-223$

$35 . \operatorname{BETA}(15,13)=-1.0$

36. $=\operatorname{BETA}(15,14)=-1.0$

37. $\operatorname{BETA}(15,22)=-\ell_{34}$

38. $\operatorname{BETA}(16,14)=-1.0$

39. $\operatorname{BETA}(16,15)=1.0$

40. $\operatorname{BETA}(16,23)=-2,4$

41. BETA $(17,15)=-1.0$

42. $\operatorname{BETA}(17,16)=1.0$

43. $\operatorname{BETA}(17,24)=-l_{56}$

44. BETA $(18,16)=-1.0$

45. BETA $(18,25)=-\ell_{67}$
45. $\operatorname{BETA}(19,17)=-1.0$

$47 . \quad \operatorname{BETA}(19,18)=1.0$

$48 . \quad$ BETA $(19,27)=-289$

$49-\operatorname{BETA}(20,18)=-1.0$

$50 \ldots$ BETA $(20,19)=1.0$

51. BETA $(20 ; 28)=-9 ; 10$

52. $\mathrm{BETA}(21,20)=-1.0$

53. BETA $(21,21)=1.0$

54. BETA $(22,21)=-1.0$

$-55=\operatorname{BECA}(22,22)=1.0$

56. $\operatorname{BETA}(23,22)=-1.0$

$57=\operatorname{BETA}(23,23)-1.0$

58. ВE'TA $(24,23)=-1.0$

59. $\operatorname{BETA}(24,24)=1.0$

E0. В̈ЕTA $(25,24)=-1.0$

61. ВE'ГA $(25,25)=1.0$

62. BETA $(26,25)=-1.0$

63. $\operatorname{BETA}(26 ; 26)=1.0$

64. BETA $(27,27)=-1.0$

65. $\operatorname{BETA}(27,28)=1.0$

66. BETA $(28,28)=-1.0$

67. $\operatorname{BETA}(28,29)=1.0$

68. $\operatorname{BETA}(29,7)=-1.0$

69. $\operatorname{BETA}(29,11)=1.0$

The [K] matrix is set up as follows from Ref. 2.

1. $A I I K_{i j}=0$ except for those that follow $\left(K_{i j} \equiv \operatorname{KAPPA}(i, j)\right)$

2. $\operatorname{KAPPA}(1,1)=\hat{A}_{12} \mathrm{E}_{\mathrm{R}} / \ell_{12}$ 
3. $\operatorname{KAPPA}(2,2)=A_{23} E_{R} / 2{ }_{23}$

4. KAPPA $(3,3)=A_{34} E_{R} / l$

5. $\operatorname{KAPPA}(4,4)=A_{45} E_{R} / 45$

6. $\operatorname{KAPPA}(5,5)=A_{56} E_{R} /{ }^{0} 56$

7. $\operatorname{KAPPA}(6,6)=A_{67} E_{R} / l_{67}$

8. $\operatorname{KAPPA}(7,7)=\mathrm{K}_{28}$

9. $\operatorname{KAPPA}(8,8)=\mathrm{K}_{38}$

10. $\operatorname{KAPPA}(9,9)=A_{89} E_{c} / s_{89}$

11. KAPPA $(10,10)=A_{9,10} E_{c} / 8,10,9$

12. $\operatorname{KAPPA}(11,11)=\mathrm{k}_{10,5}$

13. $\mathrm{KAPPA}(12,12)=\mathrm{K}_{10,6}$

14. $\operatorname{KAPPA}(13,13)=12 \mathrm{E}_{\mathrm{R}} \mathrm{I}_{12} / 23$

15. $\operatorname{KAPPA}(13,21)=-6 \mathrm{E}_{\mathrm{R}} \mathrm{I}_{12} / \ell_{12}$

16. $\operatorname{KAPPA}(14,14)=12 \mathrm{E}_{\mathrm{R}} \mathrm{I}_{23} / \ell_{23}^{3}$

17. $\operatorname{KAPPA}(14,22)=-6 \mathrm{E}_{\mathrm{R}} \mathrm{I}_{23} / \ell 23$

18. $\operatorname{KAPPA}(15,15)=12 \mathrm{E}_{\mathrm{R}} \mathrm{I}_{34} / \ell_{34}^{3}$

19. $\operatorname{KAPPA}(15,23)=-6 \mathrm{E}_{\mathrm{R}} \mathrm{I}_{34} / \ell_{34}^{2}$

20. $\operatorname{KAPPA}(16,16)=12 \mathrm{E}_{\mathrm{R}} \mathrm{I}_{45} / \ell_{45}^{3}$

21. $\operatorname{KAPPA}(16,24)=-6 \mathrm{E}_{\mathrm{R}} \mathrm{I}_{45} /{ }^{2}{ }_{45}^{2}$

22. KAPPA $(17,17)=12 \mathrm{E}_{\mathrm{R}} \mathrm{I}_{56} / l_{56}^{3}$

23. $\operatorname{KAPPA}(17,25)=-6 \mathrm{E}_{\mathrm{R}} \mathrm{I}_{56} / \ell_{56}^{2}$

24. $\operatorname{KAPPA}(18,18)=12 \mathrm{E}_{\mathrm{R}} \mathrm{I}_{67} / \ell_{67}^{3}$

25. $\operatorname{KAPPA}(18,26)=-6 \mathrm{E}_{\mathrm{R}} \mathrm{I}_{67} / \ell_{67}^{2}$

26. $\operatorname{KAPPA}(19,19)=12 \mathrm{E}_{\mathrm{c}} \mathrm{I}_{89} / \ell_{89}^{3}$

27. $\operatorname{KAPPA}(19,27)=-6 \mathrm{E}_{\mathrm{c}} \mathrm{I}_{89} /{ }_{89}^{l} 2$

28. $\operatorname{KAPPA}(20,20)=12 \mathrm{E}_{\mathrm{c} \mathrm{I}} \mathrm{I}_{9,10} / \ell_{9,10}^{3}$ 


$$
\begin{aligned}
& \text { 29. } \operatorname{KAPPA}(20,28)=-6 \mathrm{E}_{c} \mathrm{I}_{9,10^{/ 2}, 2}{ }_{9,10} \\
& 30 . \quad \operatorname{KAPPA}(21,13)=-6 \mathrm{E}_{\mathrm{R}} \mathrm{I}_{12} / \ell{ }_{12} \\
& \text { 31. } \quad \operatorname{KAPPA}(21,21)=4 \mathrm{E}_{\mathrm{R}} \mathrm{I}_{12}^{/ 2}{ }_{12} \\
& \text { 32. } \quad \operatorname{KAPPA}(22,14)=-6 \mathrm{E}_{\mathrm{R}} \mathrm{I}_{23} / 2{ }_{23} \\
& \text { 33. } \quad \operatorname{KAPPA}_{(22,22)}=4 \mathrm{E}_{\mathrm{R}} \mathrm{I}_{23} / \mathrm{l}_{23} \\
& \text { 34. } \operatorname{KAPPA}(23,15)=-6 \mathrm{E}_{\mathrm{R}} \mathrm{I}_{34} / \ell_{34}{ }^{2} \\
& \text { 35. } \quad \text { KAPPA }(23,23)=4 \mathrm{E}_{\mathrm{R}} \mathrm{I}_{34} / l_{34} \\
& \text { 36. KAPPA }(24,16)=-6 \quad \mathrm{E}_{\mathrm{R}} \mathrm{I}_{45} / \ell{ }_{45}^{2} \\
& \text { 37. KAPPA }(24,24)=4 E_{R} I_{45} / l_{45} \\
& \text { 38. KAPPA }(25,17)=-6 E_{R} I_{56} / \ell_{56}^{2} \\
& \text { 39. KAPPA }(25,25)=4 \mathrm{E}_{\mathrm{R}} \mathrm{I}_{56} / \ell_{56} \\
& \text { 40. KAPPA }(26,18)=-6 \mathrm{E}_{\mathrm{R}} \mathrm{I}_{67} / \ell_{67}{ }^{2}
\end{aligned}
$$

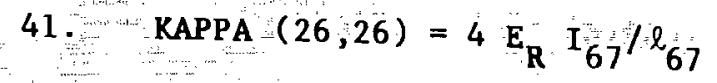

$$
\begin{aligned}
& \text { 42. } \quad \operatorname{KAPPA}(27,19)=-6 \mathrm{E}_{\mathrm{c}} \mathrm{I}_{89} / \ell_{89}^{2} \\
& \text { 43. } \quad \operatorname{KAPPA}(27,27)=4 \mathrm{E}_{\mathrm{c}} \mathrm{I}_{89} / l_{89} \\
& \text { 44. KAPPA }(28,20)=-6 E_{c} I_{9,10^{/ \ell}}{ }_{9,10}^{2} \\
& \text { 45. } \quad \mathrm{KAPPA}(28,28)=4 \mathrm{E}_{\mathrm{c}} \mathrm{I}_{9}, 10^{/ l_{9}, 10} \\
& \text { 46. } \quad \operatorname{KAPPA}(29,29)=K_{7,11}
\end{aligned}
$$

The $29 \times 29$ damping and stiffness matrices are computed from:

$$
\begin{aligned}
& {[\mathbf{C}]=\left[{ }_{\beta} \mathbf{T}\right][\gamma][\beta],} \\
& {[K]=\left[\beta^{T}\right][K][\beta],}
\end{aligned}
$$

where $\left[\beta^{T}\right]$ is the transpose of $[\beta]$. 
$\mathrm{K}(29,29)$ will be the nonlinear stiffness of the coupler $k, 11$. In the solution of the equations of motion $(A-1)$ at each time step, $u_{7}, 11$ is evaluated and the data of Fig. 2 of the main report are used in tabular form for the appropriate $k_{7,1}$ This value $\left(u_{7,11}\right)$ if different, is substituted into the $[K]$ matrix appropriately and iterated.

A Runge-Kutta numerical integration procedure was used in the digital code. The code and subroutines appropriate to this are presented in Appendix C. The initial input velocity $v_{1}$ was assigned to ${ }^{\circ}$ through $u_{10}$ The resistance force $R_{F}$, which was taken to be 0.2 times the weight of mass eleven, was assigned to $F$ (11) as

$\mathbf{F}(11)=-\mathbf{R}_{\mathbf{F}}$

There are two other cases that can be simulated by RICTL. These are

(1) The railcar striking train free to rol1, in this case $R_{F}=0$, and

(2) A train striking the railcar free to roll, in this case $u_{i}$ through $\dot{u}_{10}$ are zero and $\dot{u}_{11}=-\dot{v}_{1}$, and $R_{F}=0$. 


\section{APPENDIX C}

$=$

COMPUIER LISTING OF RICTL

Wal. Identification No. 1.P-0880

5
$=$
5
5
$=$
$\vdots$
$\vdots$
$\vdots$
$\vdots$

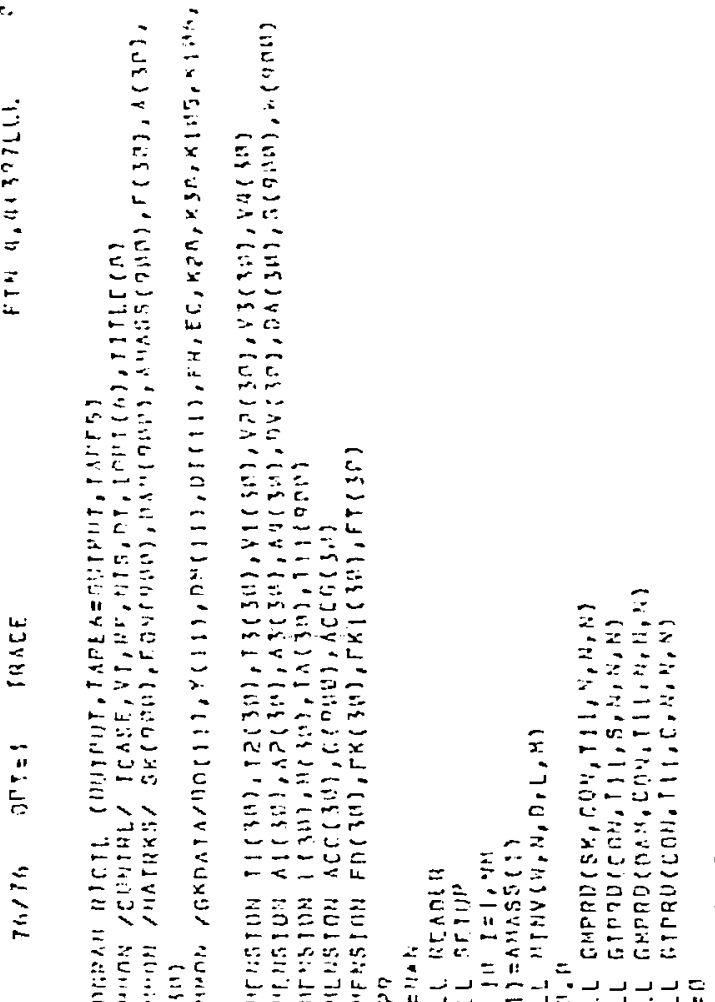




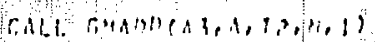

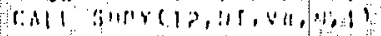

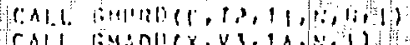

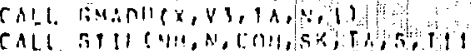

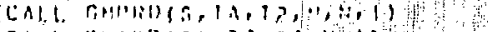

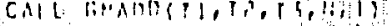

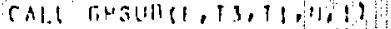

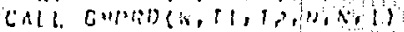

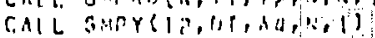

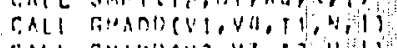

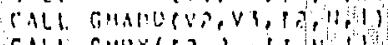

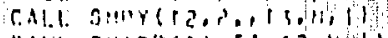

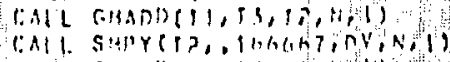

coli grantre

Call Gand al,

Cail semycis

cul cainne

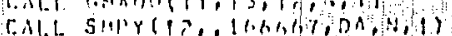

call Colnhlo, iv; $x, y, 1)$

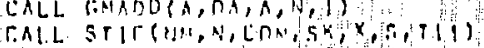

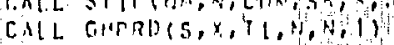

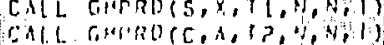

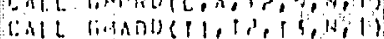

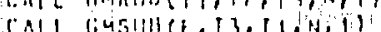

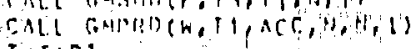

$T=1+n\}$

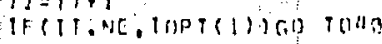

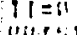

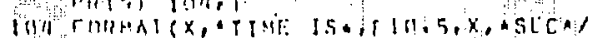
millig inj

14ri FuKHAT AC( $1(1)=A(5)(1)<5$

319 Pla III $1(13,1, x(1), A(1), A c c(1)$

113 $F$ UIIIA $1(x, 15,515, \pi, 5,12,15,115,7)$

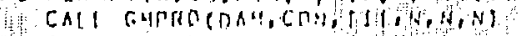

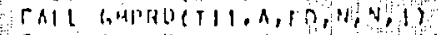

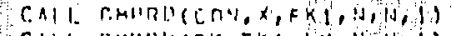

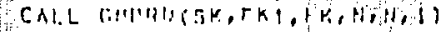

no 5 (n) $1=1, ?$

(1) $r(1)=5 k(1)+513(d)$

कीजा 16?

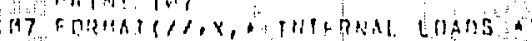

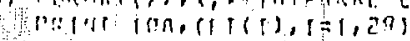

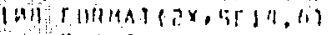

entill 1 Hh

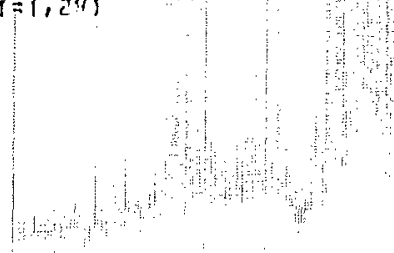




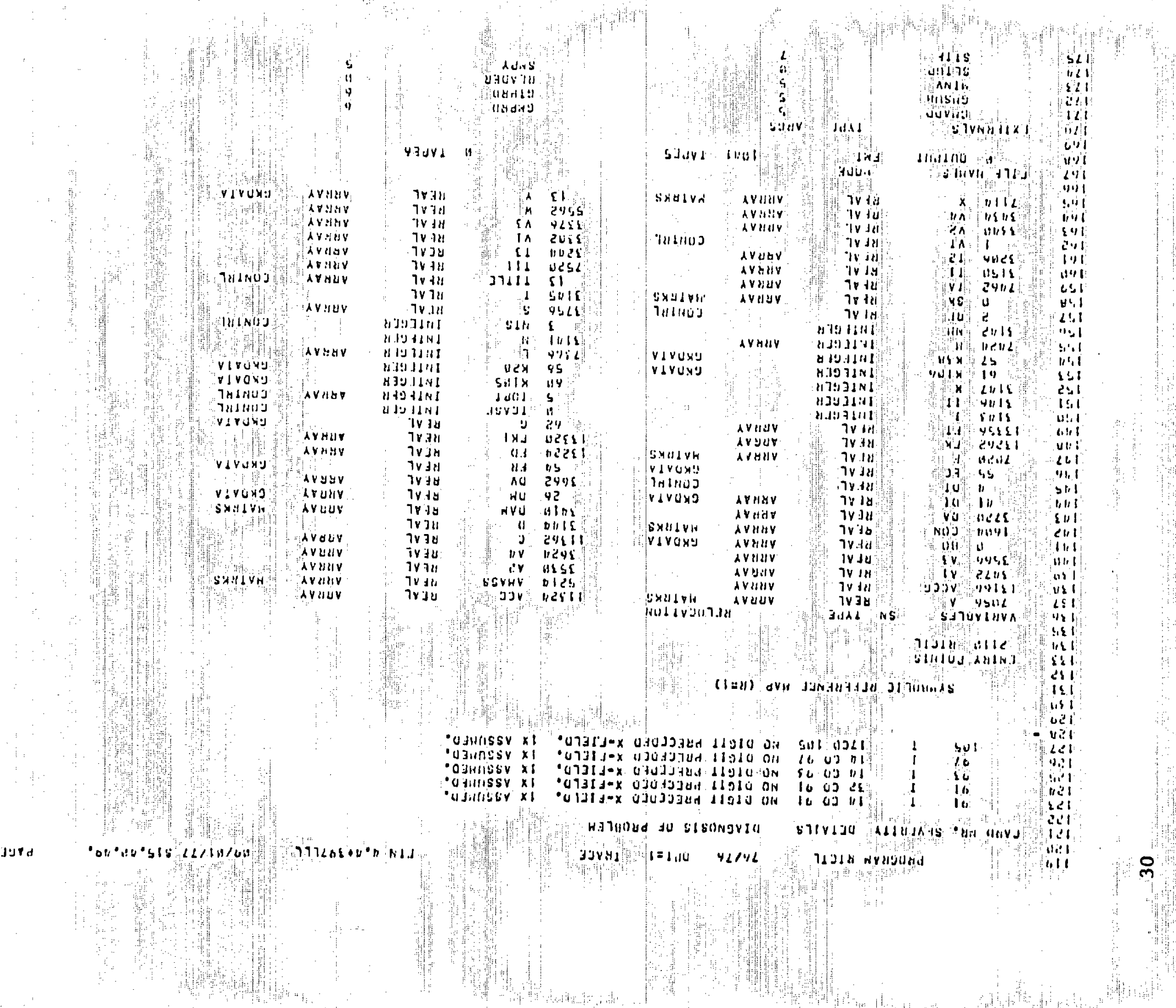


訔

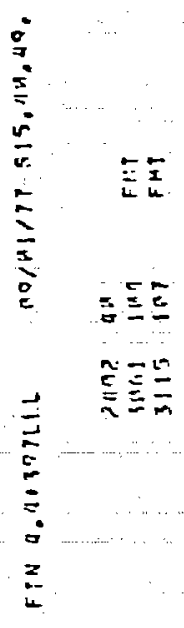

$\frac{5}{\bar{\Sigma}}$

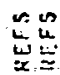

突

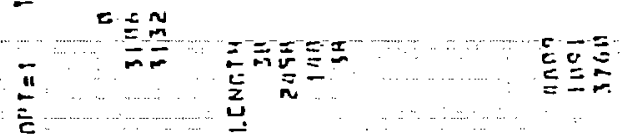

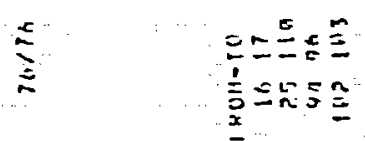

$\equiv \equiv$

新

$\underline{5}$

$\frac{x}{\bar{z}}$

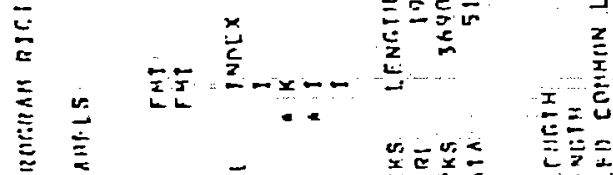

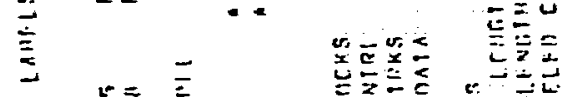

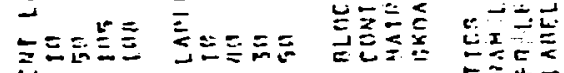

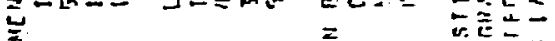

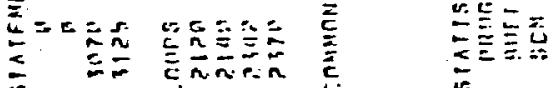

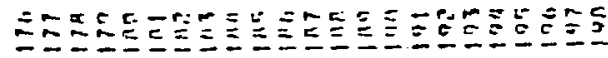




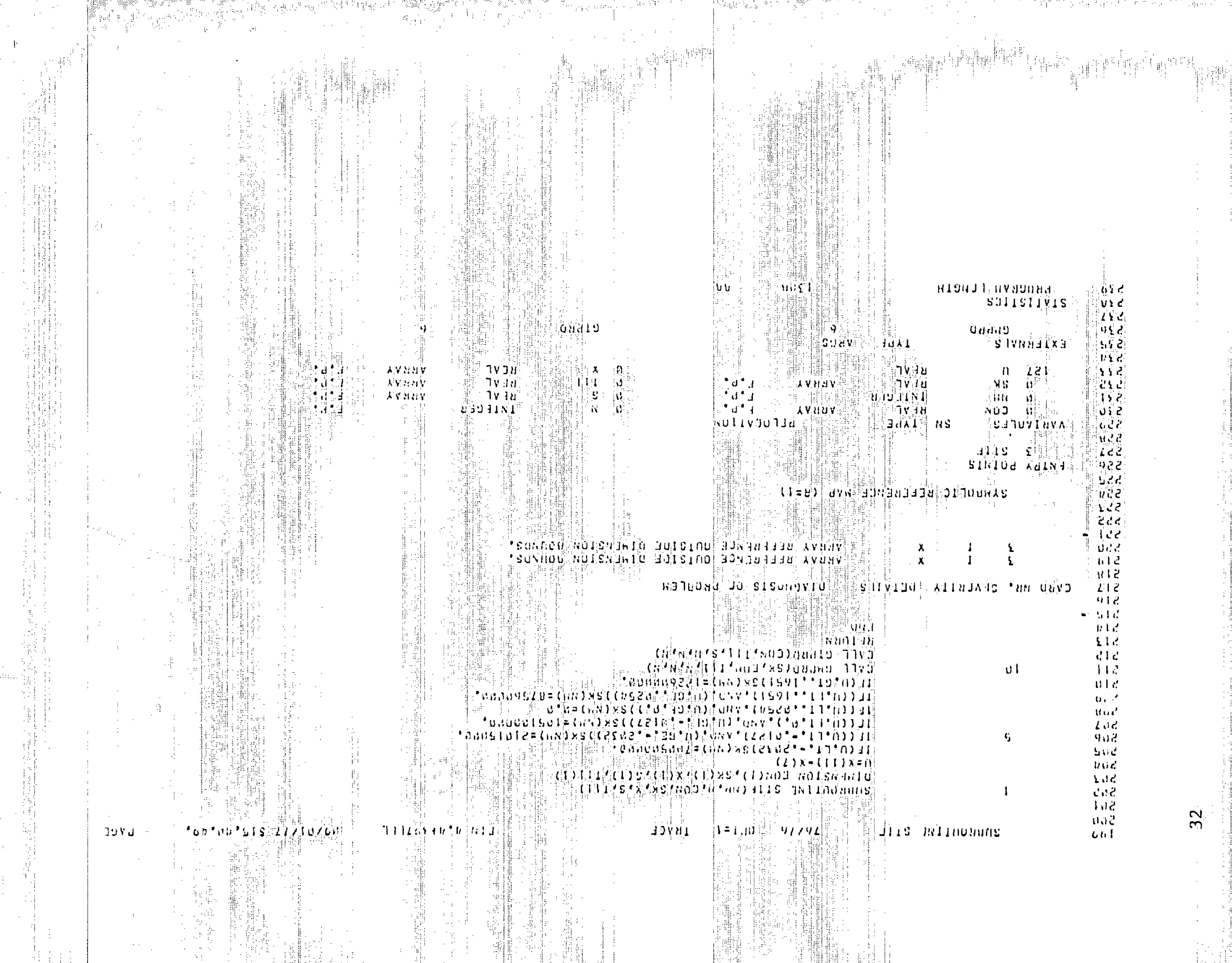



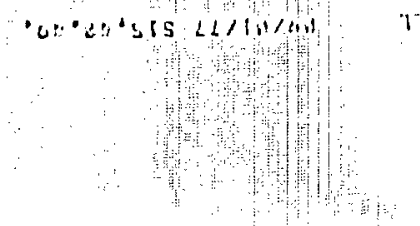


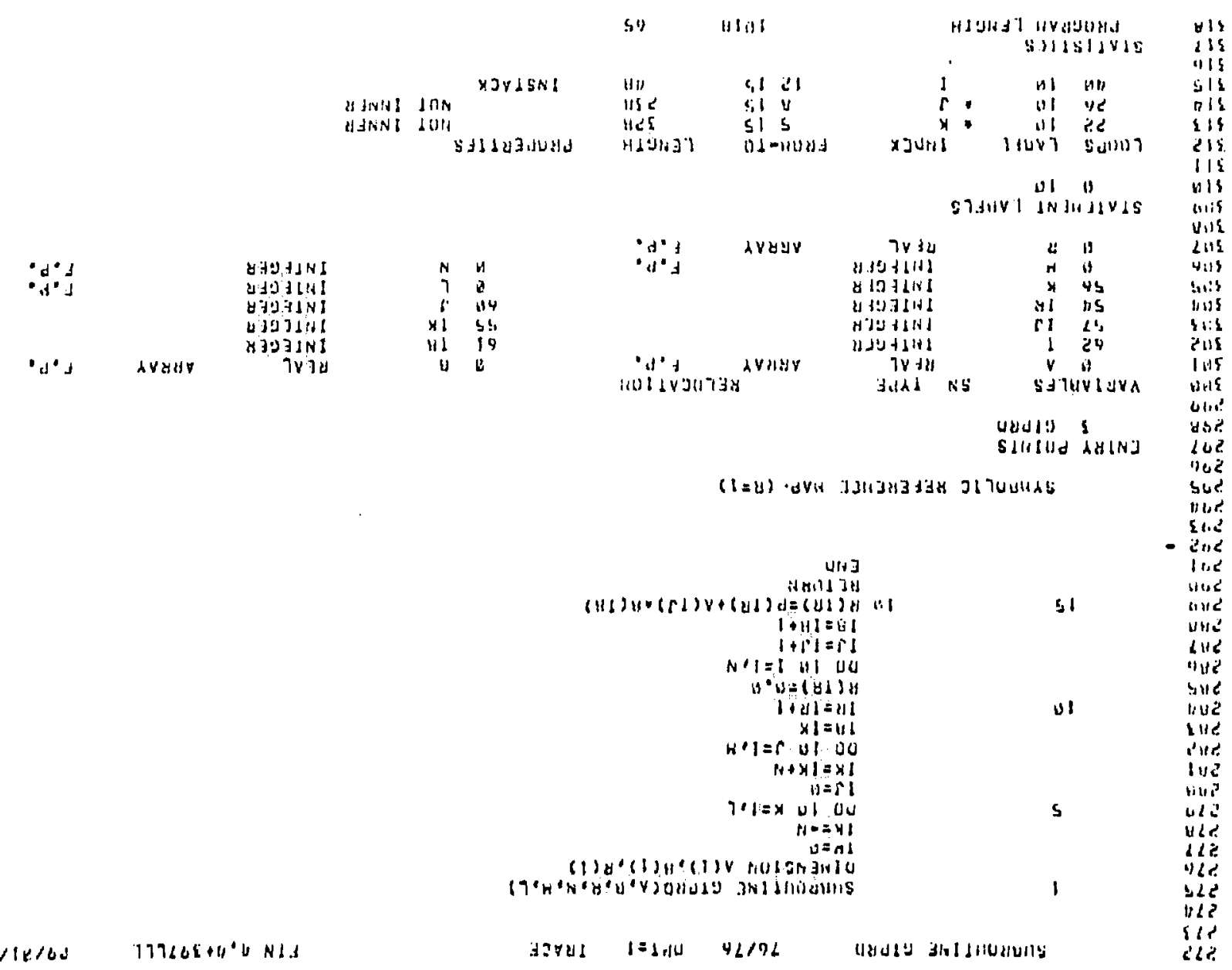




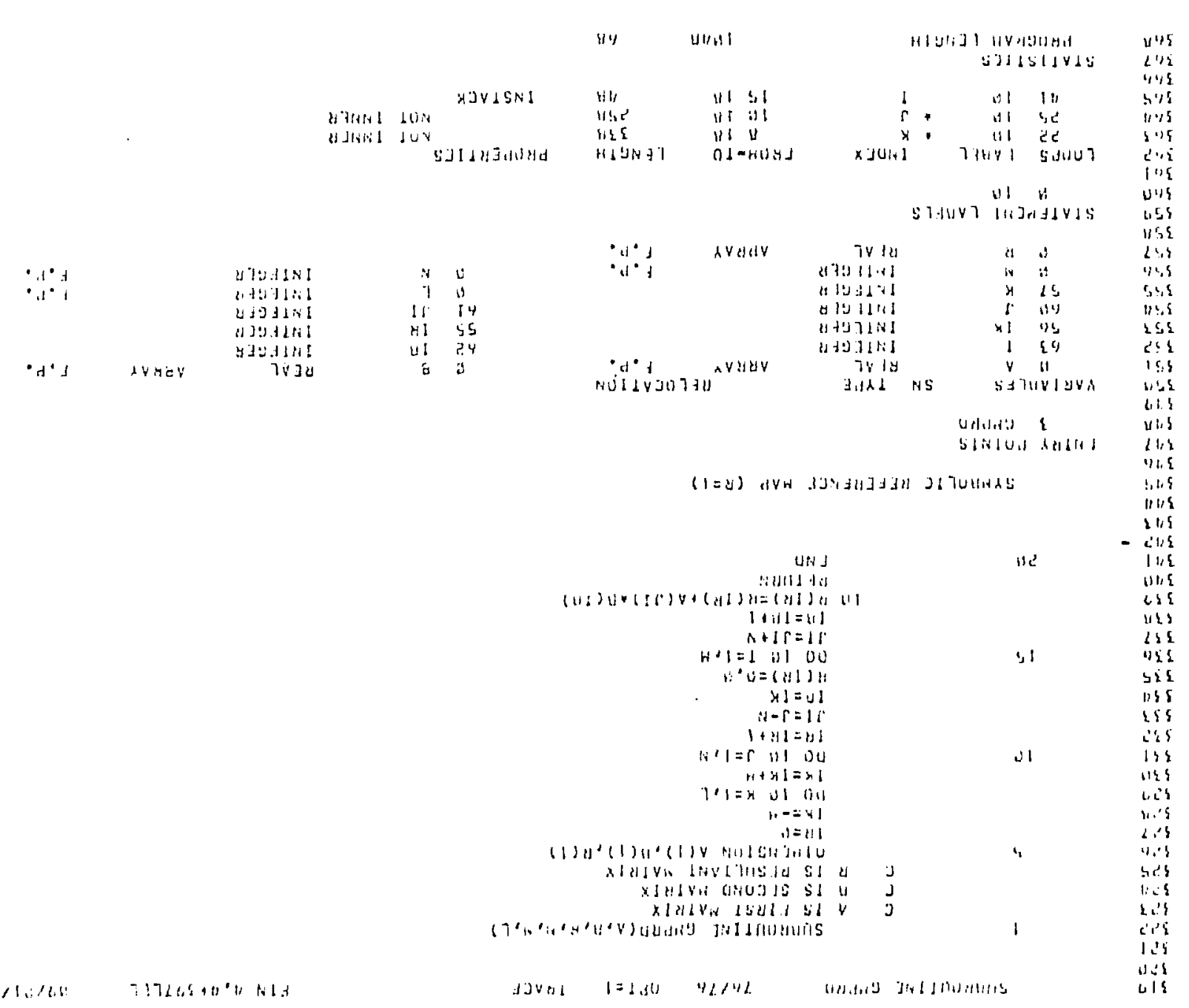




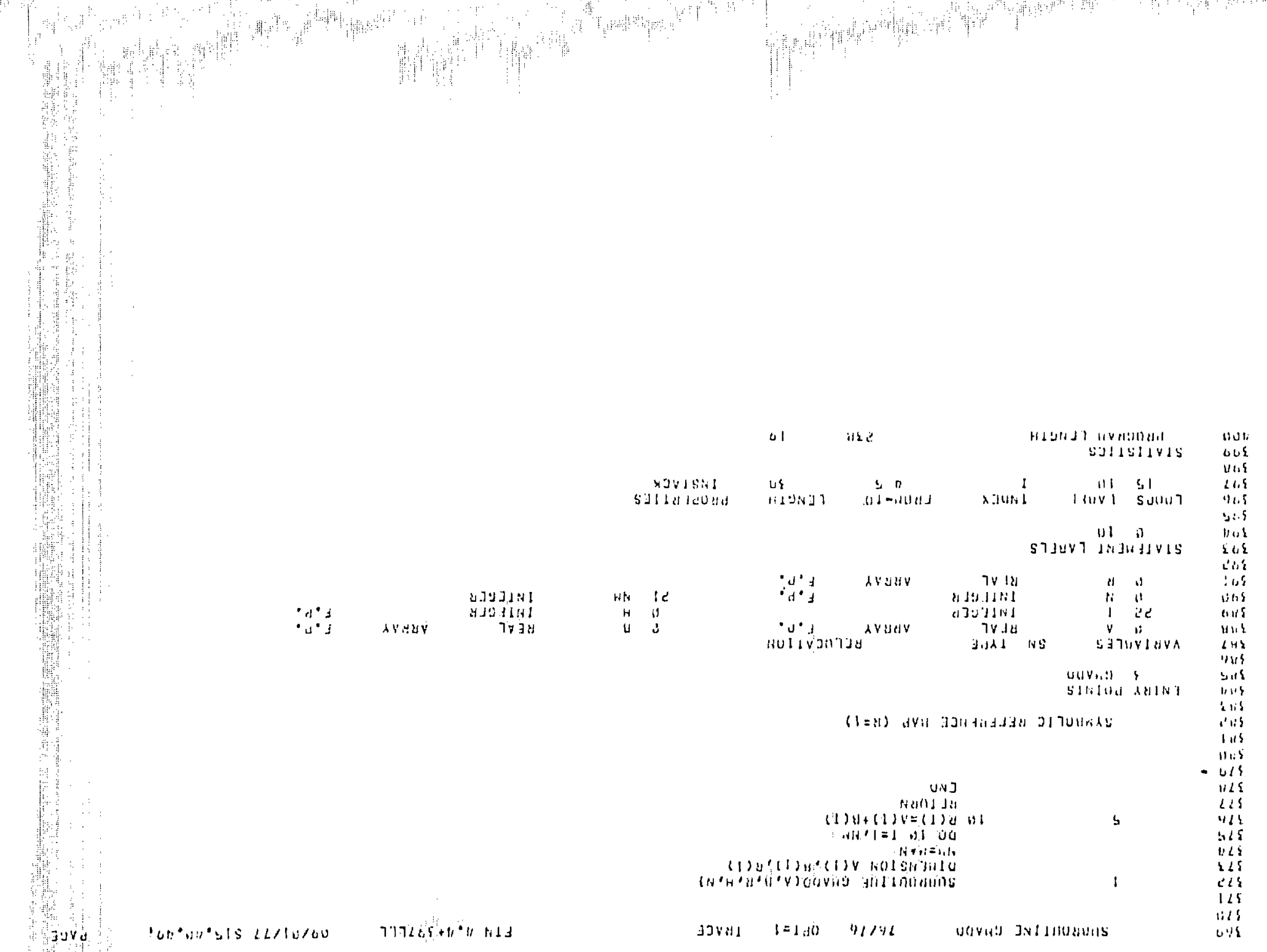




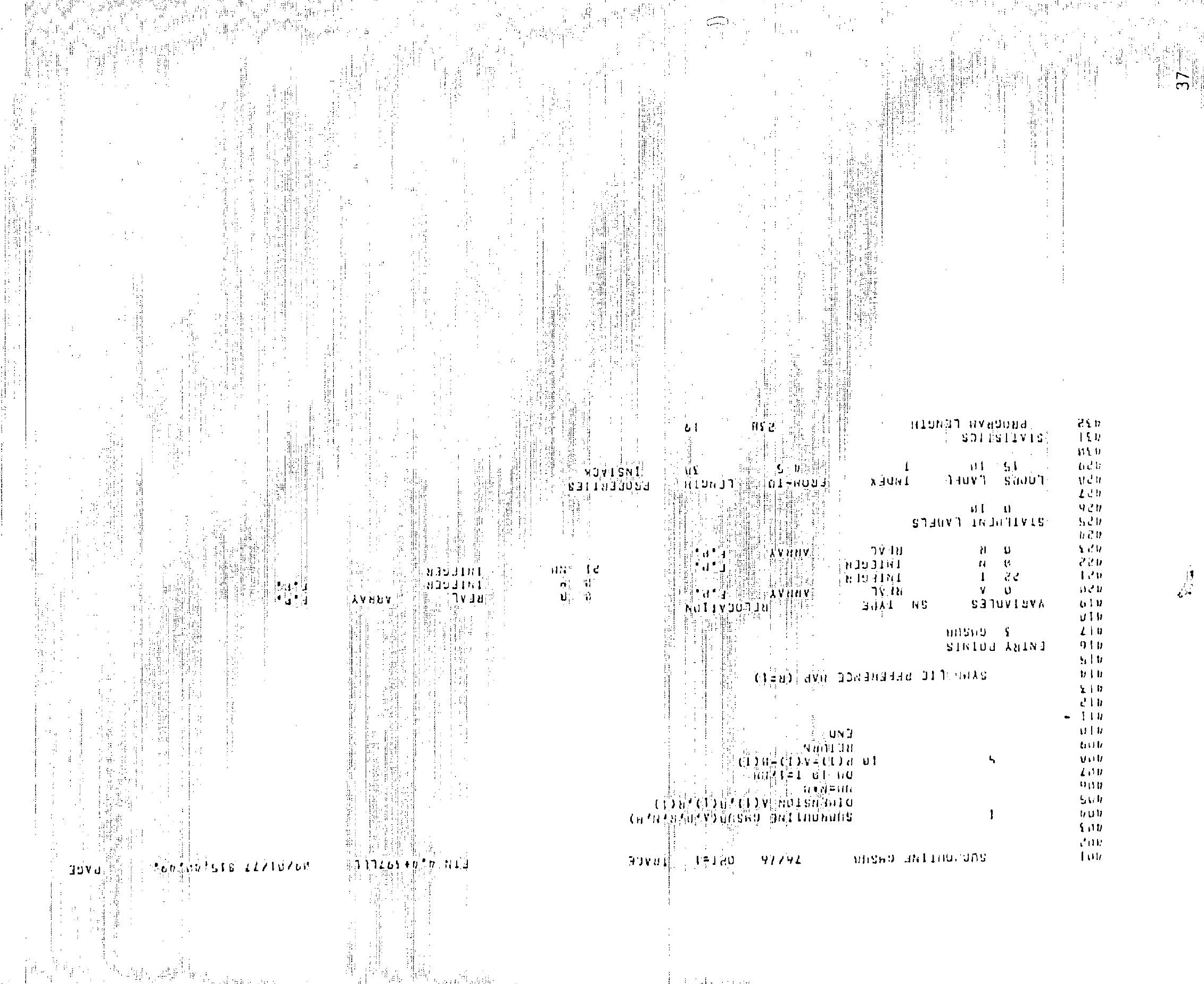


$\vdots$
$\vdots$
$\vdots$
$\vdots$
$\vdots$
$\vdots$

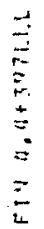

$\leftarrow x \leftarrow \simeq \simeq x$ 远岕岕导心

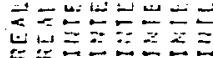

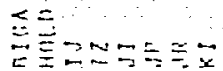

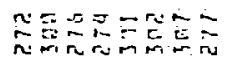

$\stackrel{\breve{\Xi}}{\Xi}$

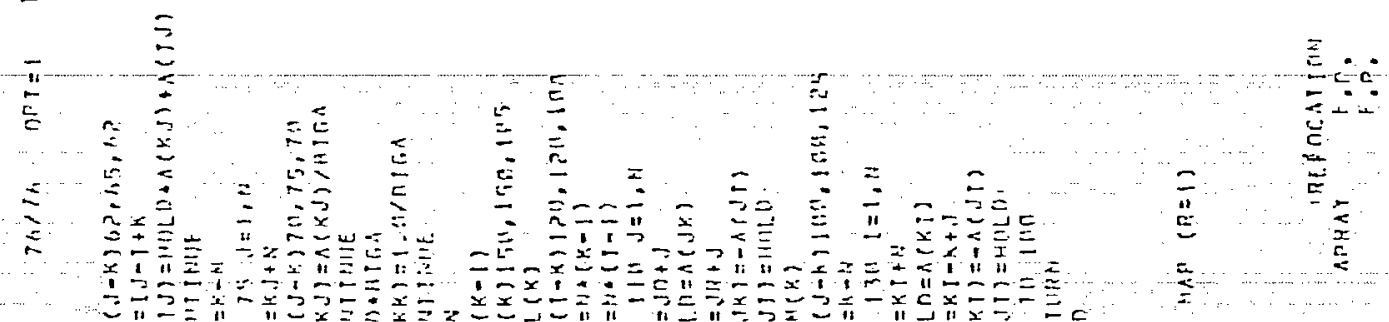

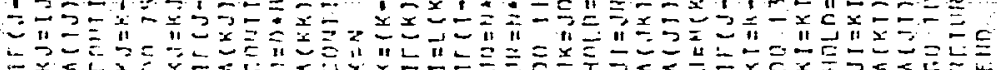

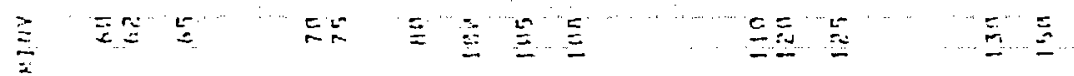

产

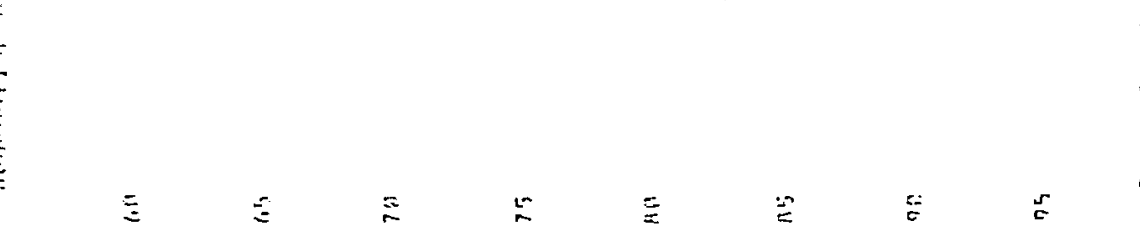

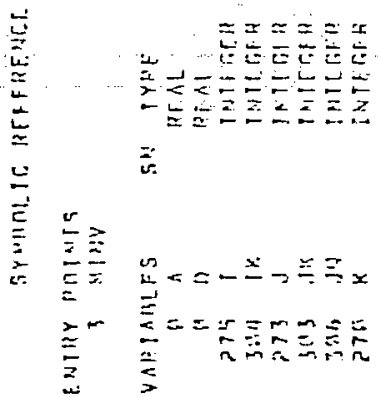

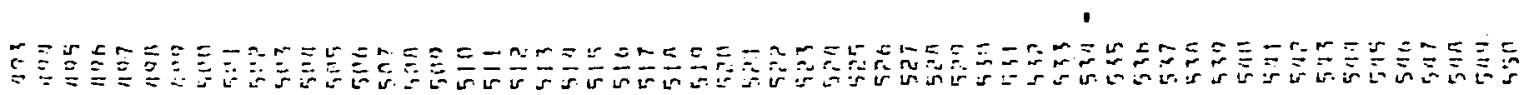




\section{巳ே:}

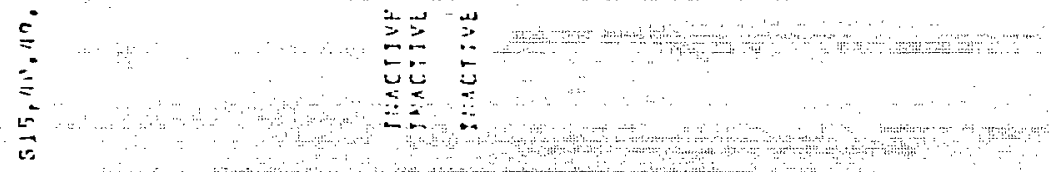

and

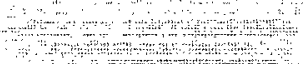

2

$z$
$a$

$+2$

\begin{tabular}{ll} 
& \\
\hline & $=$
\end{tabular}

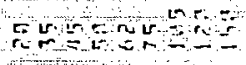

it

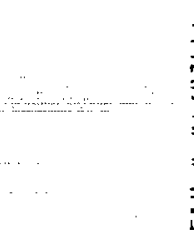

$-2=2$

2

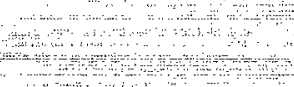

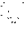

and $==2$

$=$

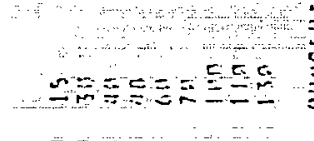

$$
a=c_{r}=\frac{\pi}{n}=5
$$

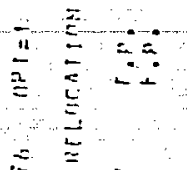

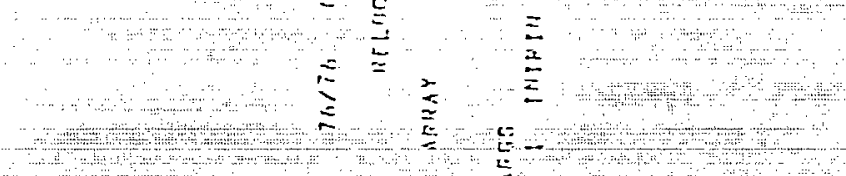

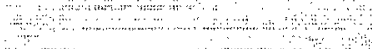

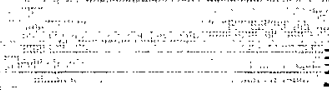

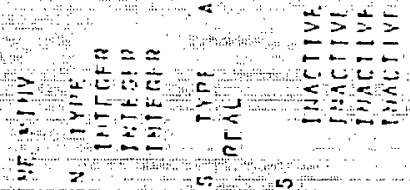

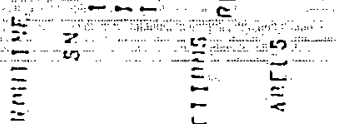

产

8

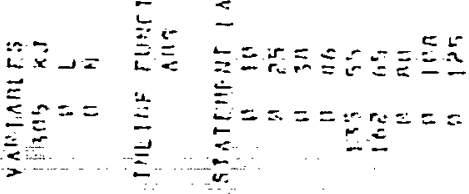

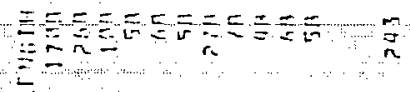

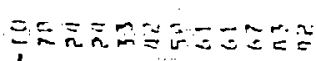

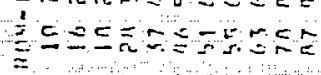

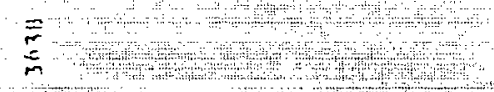

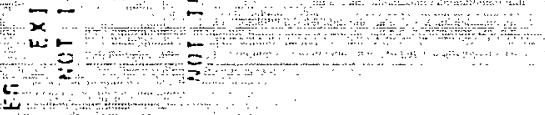

xs

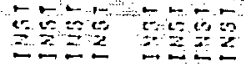

$25+3+3=3$

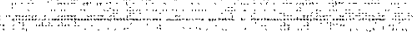

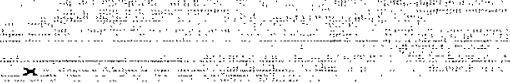

$\stackrel{2}{5}$ $-x-m-x-2=-3$

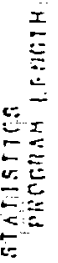

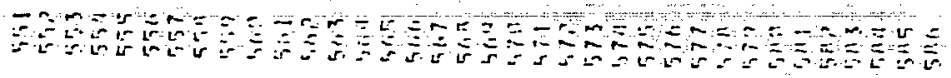


บขe

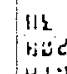

bicic

Hing

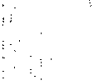

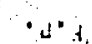

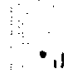

$\bullet, 1 \cdot$

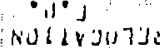

$\therefore \quad 10$

$0: 17$

nl-iduld
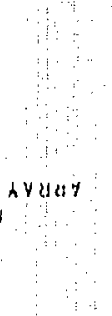

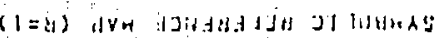

UNi

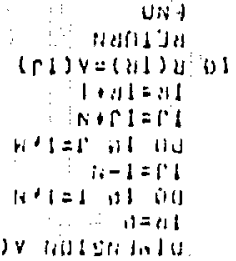

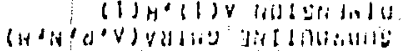

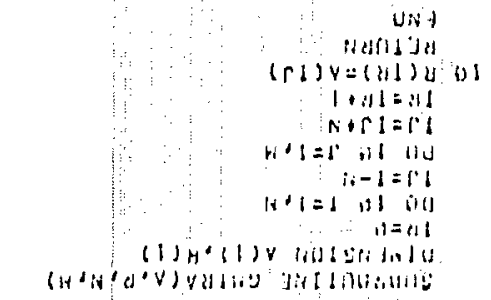

J.2ทा

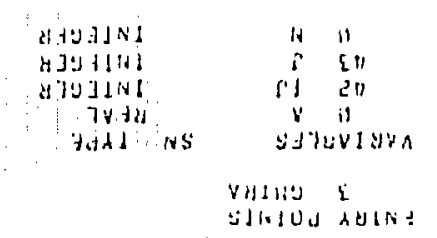

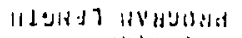 แIIISI I I \\ 11! 118

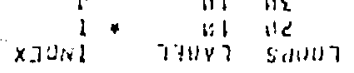

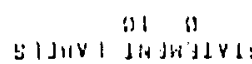

muras

$i=6,10$

$4 / 2116$

Vulive disldumblls [a?

(2)

(12)

पid

(11)

all

1114

21

(i)

ט।1?

und,

पiv!

tim

t....

bis
ris

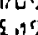

Sil

- IJ"

iin'

(ii)

this

nilis

in:

ints

ins

$3+5$
16,4

1) 14.6

o:15

जाi 

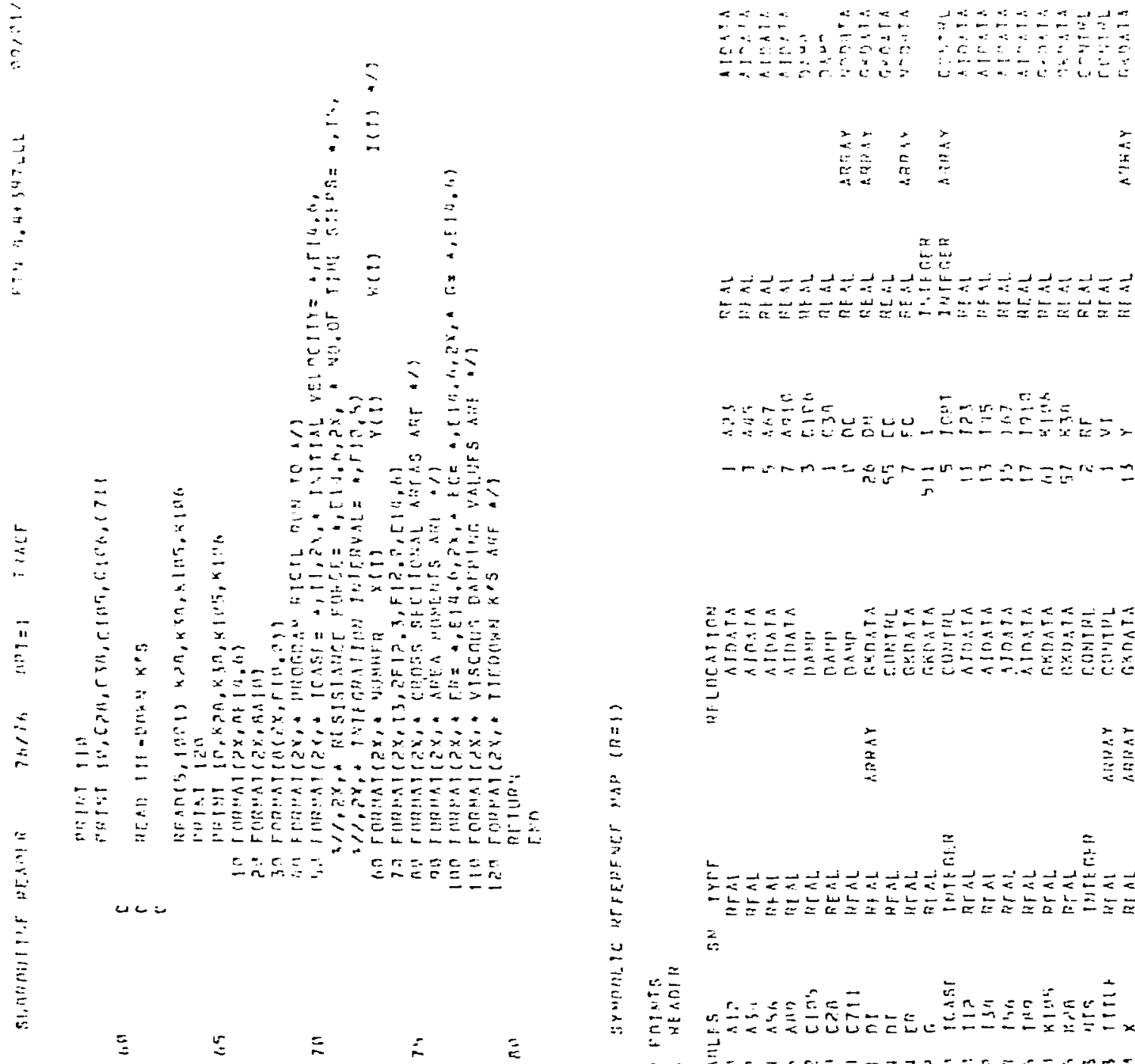

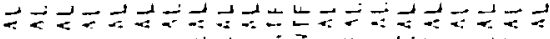

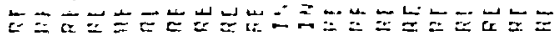

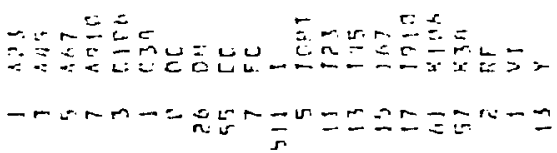

过

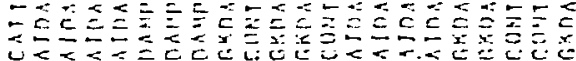

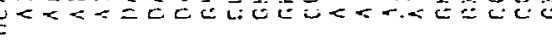

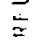

$\underset{x}{x}$

2
$\vdots 2$
$\vdots$

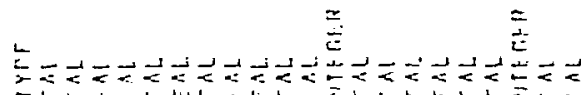

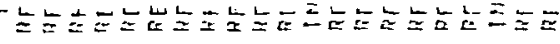
$\stackrel{2}{2}$

这

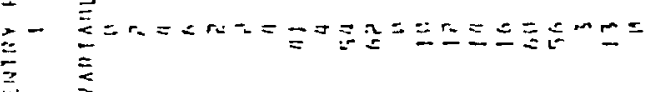

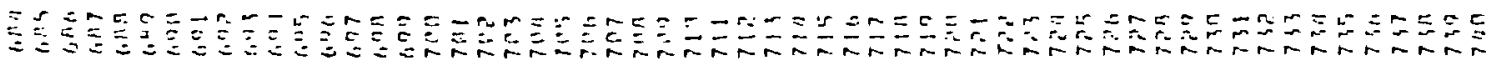




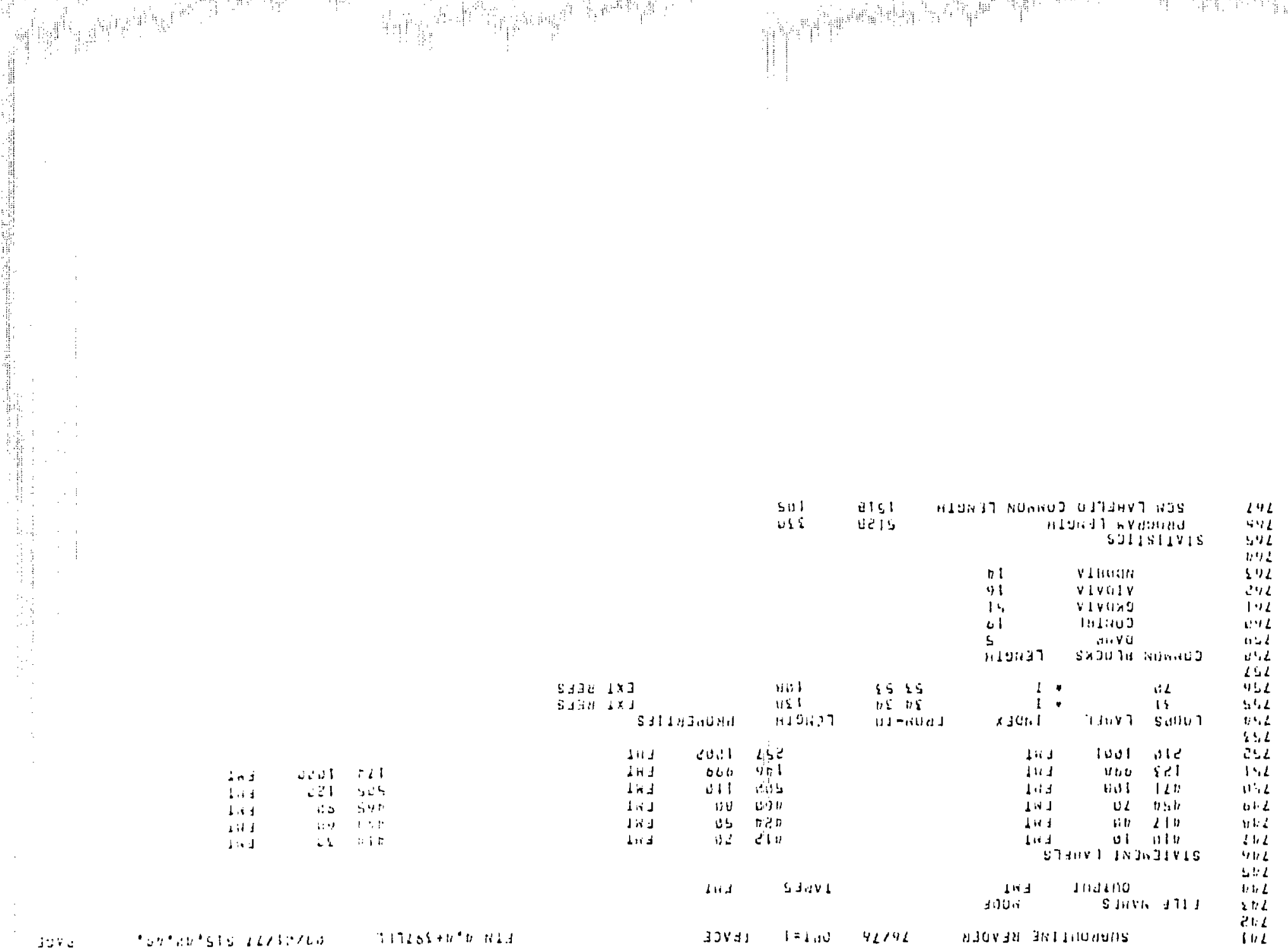




\begin{tabular}{|c|c|c|c|}
\hline \multirow{2}{*}{\multicolumn{4}{|c|}{ 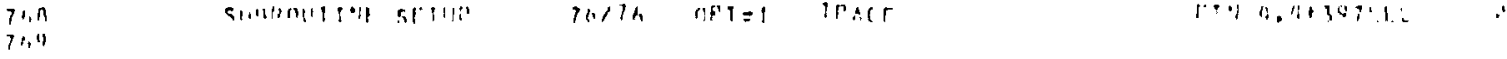 }} \\
\hline & & & \\
\hline 171 & 1 & & 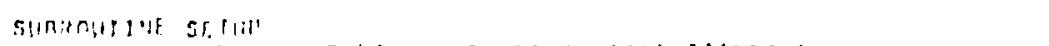 \\
\hline יו & & & 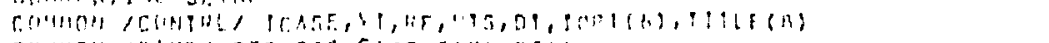 \\
\hline 373 & & & 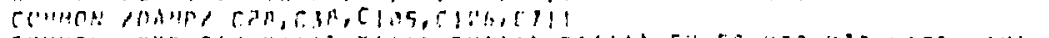 \\
\hline 711 & & & 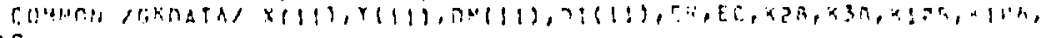 \\
\hline 371 & 5 & & 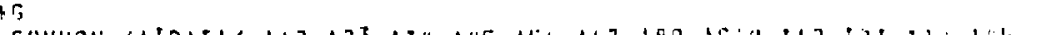 \\
\hline $71 \%$ & & & 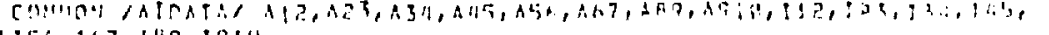 \\
\hline 717 & & & $1154,1+7,1 k 9,1819$ \\
\hline$i \ln$ & & & 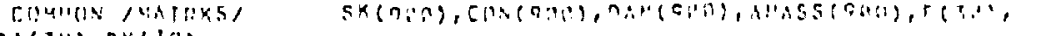 \\
\hline 771 & & & $1 A(36), n \times(36)$ \\
\hline 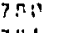 & 1" & & 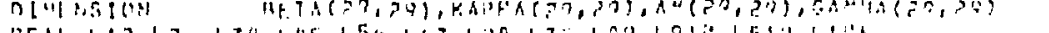 \\
\hline $7 n !$ & & & 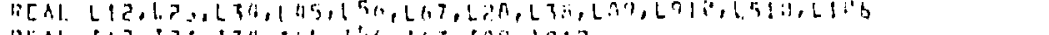 \\
\hline $17 \because$ & & & 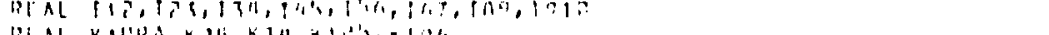 \\
\hline l113 & & & 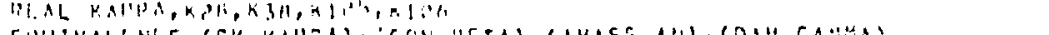 \\
\hline $7 ! 11$ & & & 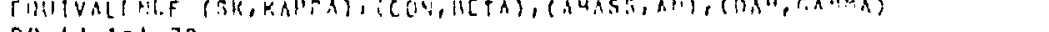 \\
\hline $7 " 1 \mathrm{i}$ & 15 & & $0 ? 1,1=1,79$ \\
\hline $7 n_{11}$ & & & n! $19.1=1,70$ \\
\hline $7 n 7$ & & & $" 1: 1 \wedge(1,1)=n$ \\
\hline $13 n$ & & & $x A \cdots+(1, j)=0$ \\
\hline $7 \mathrm{man}$ & & & $A \prime(1,1)=1$ \\
\hline $7 \cdot 311$ & ?11 & 1.1 & $\operatorname{com}(1,1)=0$ \\
\hline 711 & & & $41 \cdot=x(7)-x(1)$ \\
\hline 74) & & & $1,3=0(3)-x(3)$ \\
\hline צי 7 & & & $(1)=x(4)-1(3)$ \\
\hline 744 & & & $L 115=x(5)-x:(1)$ \\
\hline 79 & 75 & & $\begin{array}{l}1.56=x(6)-x(5) \\
1,7=x(7)-x(6)\end{array}$ \\
\hline $7 \%$ & & & 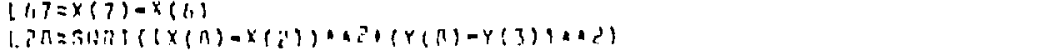 \\
\hline 107 & & & 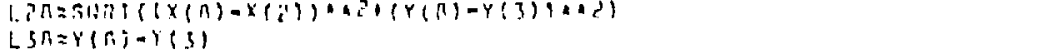 \\
\hline $7 \cdot 11$ & & & $\begin{array}{l}\operatorname{Ls} n=Y(n)-Y(3) \\
\ln n=x(n)-x(n)\end{array}$ \\
\hline $7 \mathrm{BM}$ & & & 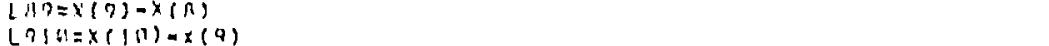 \\
\hline חיח & 36 & & $\begin{array}{l}(9) 4=x(117)-x(9) \\
1.514=y(17)-y(5)\end{array}$ \\
\hline $8(4)$ & & & 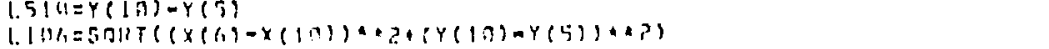 \\
\hline 1:? & & & 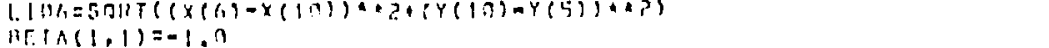 \\
\hline $\begin{array}{l}n+1 \\
n: 1 \\
n: 1 i n\end{array}$ & & & $\begin{array}{l}\operatorname{Mr} \ln (1,1)=-1,0 \\
\operatorname{Min}(1,2)=1,0^{\circ}\end{array}$ \\
\hline$n_{i}{ }_{i}$ & si & & ULin(a,r)=-1,n \\
\hline ?חי & & & at $11(2, y)=1 . \pi^{4}$ \\
\hline $1: 17$ & & & $11 ! i n(3,1)=-1, n$ \\
\hline ก(1:4 & & & $n[T, A(3, n)=1, n$ \\
\hline$n .17$ & & & $\operatorname{Br} T \operatorname{Tn}(4,1)=-1,4$ \\
\hline A16" & 116 & & $H E\{A\langle n, j\}=1, B$ \\
\hline n11 & & & nI: $T \wedge(5,5)=-1 . n$ \\
\hline$\pi$ & & & IIF $T A(5,6)=1, !$ \\
\hline$\pi ! 3$ & & & $\operatorname{III} P A(A, B)=-1, A$ \\
\hline 114 & & & $11[T A(A, 7)=1,4$ \\
\hline n!5 & 114 & & IIE $\left\{A\left(7, r^{\prime}\right)=-L ?\right\} / 2 \Omega$ \\
\hline P!! & & & 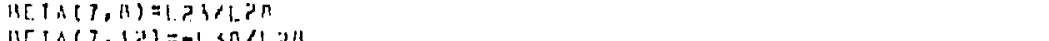 \\
\hline A17 & & & 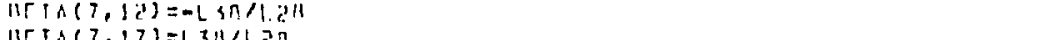 \\
\hline$\pi ! n$ & & & 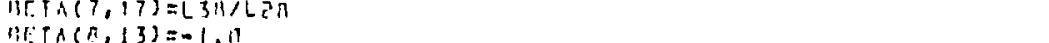 \\
\hline 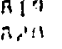 & 511 & & 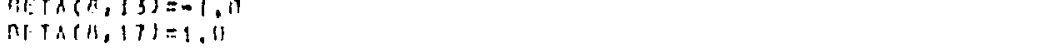 \\
\hline n, 1 & & & MC. TA(9, 19$)=-1,11$ \\
\hline$m$ & & & $111 \wedge(7,2)=1.11$ \\
\hline$n, 3$ & & & $10 \times T A(1,1,0)=-1.11$ \\
\hline " & & & $\|[T A(11,1,14)=1$, , \\
\hline$n$ & 75 & & $14[1 \mathrm{~A}(11,14)=-i, 1)$ \\
\hline 11,24 & & & $\operatorname{III} P A(11,19)=1.01$ \\
\hline דיהח & & & 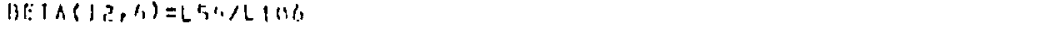 \\
\hline
\end{tabular}


岕

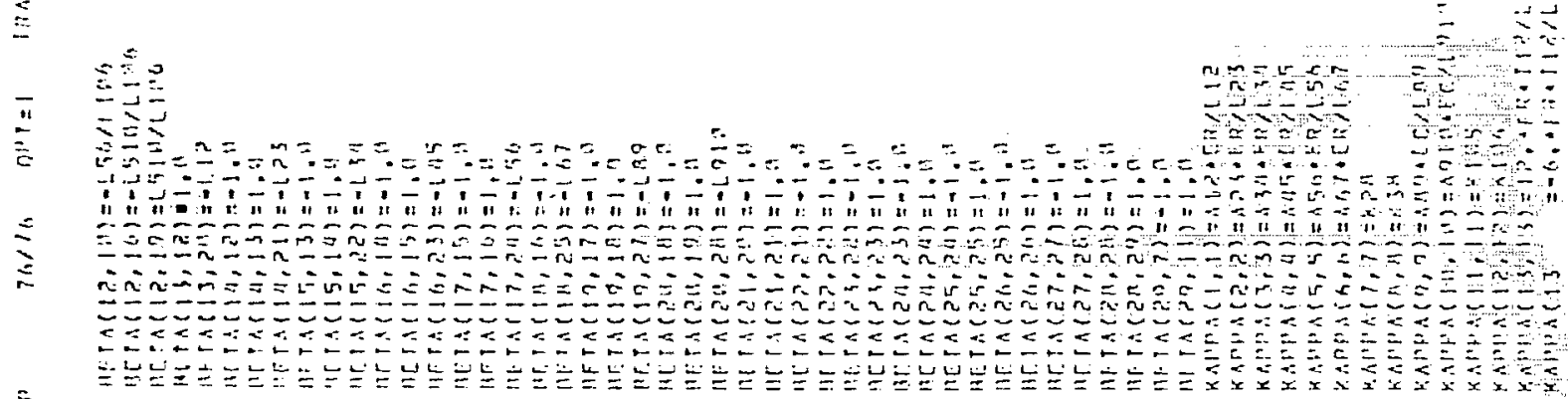

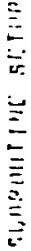

$\equiv \quad \vdots$

$=$

$\stackrel{E}{E}$

$\bar{c}$

$\Xi$

เே

$\underline{\underline{E}}$

$\underline{5}$

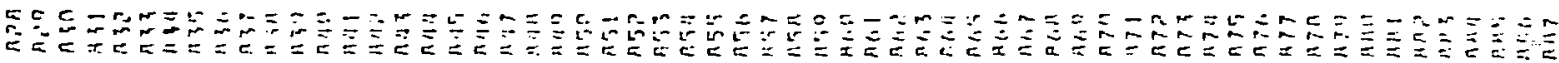




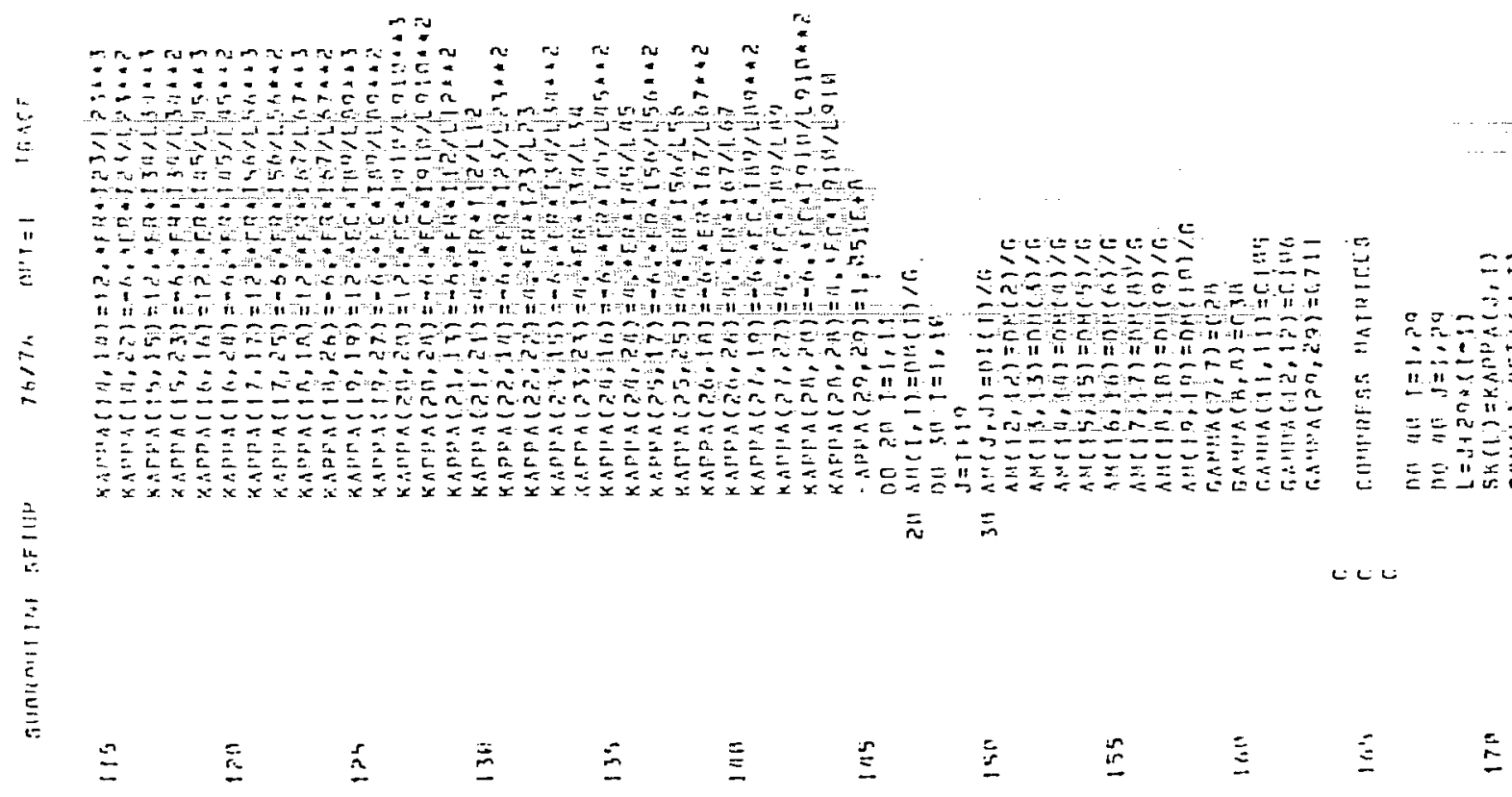

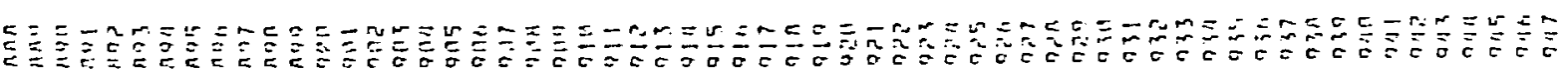




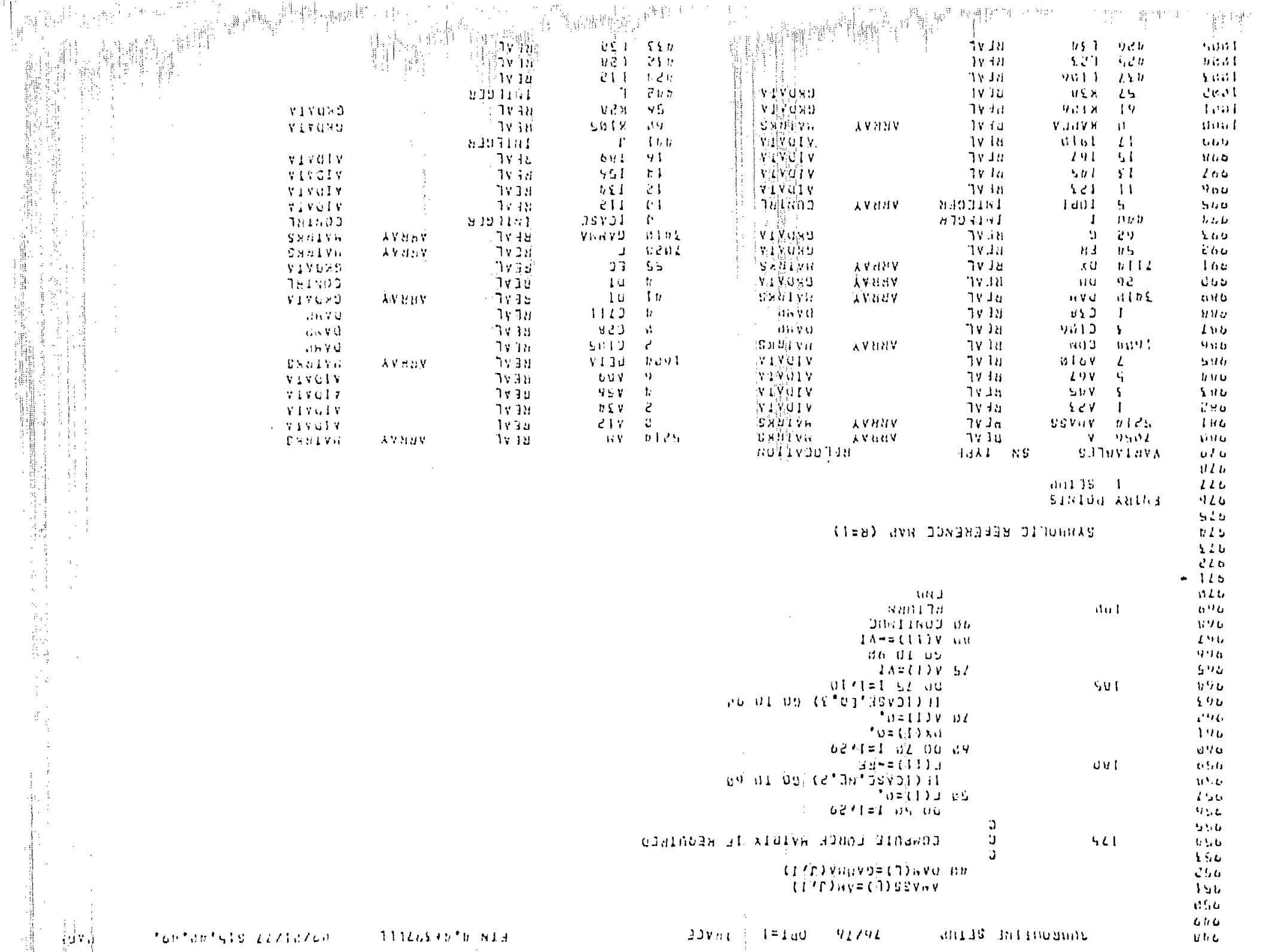




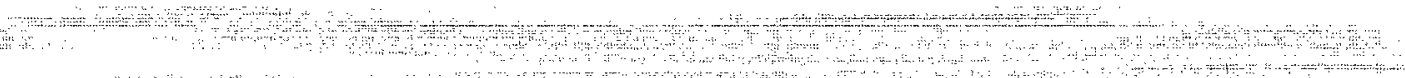
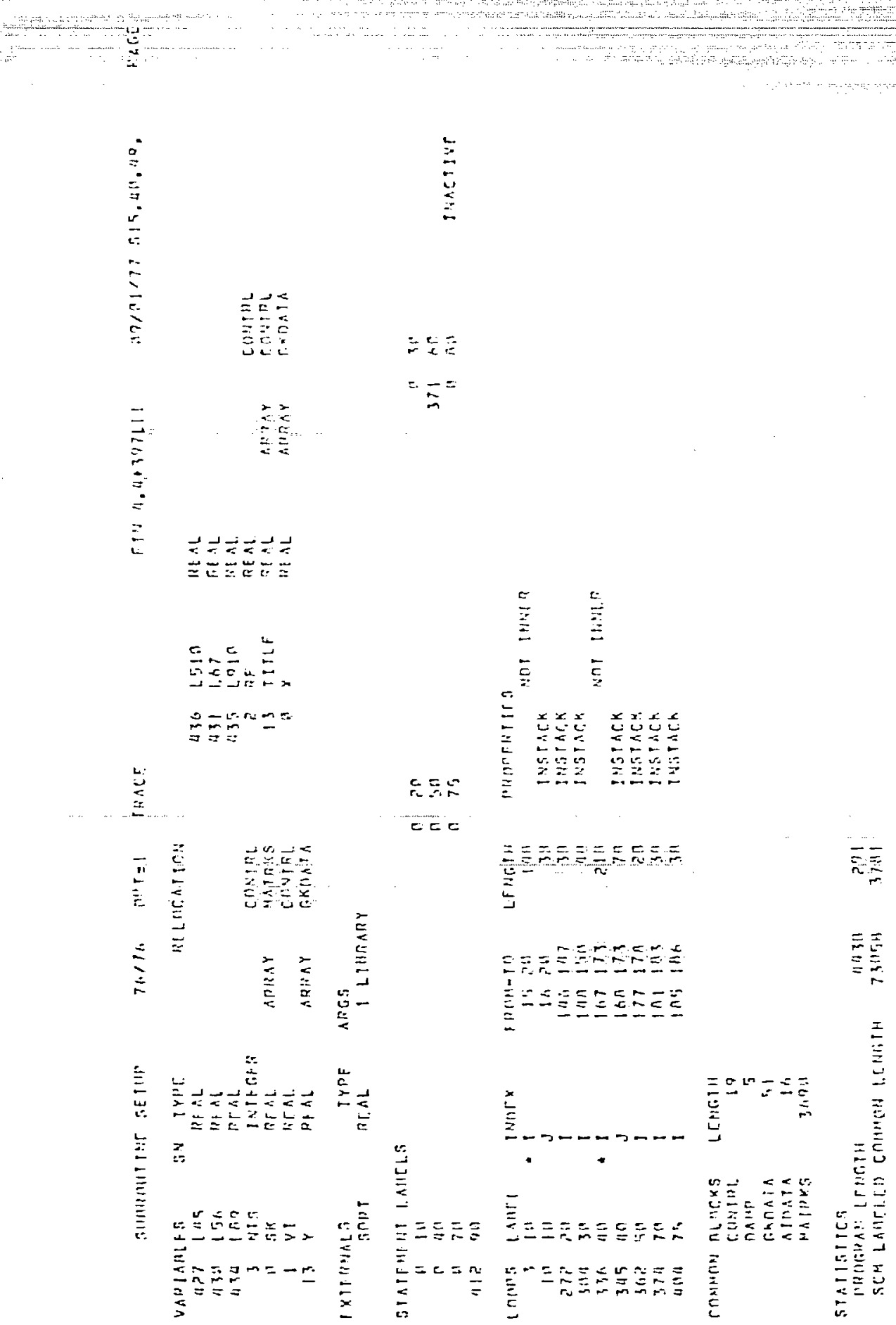

Hent 
$\stackrel{\Xi}{\vdots}$

and

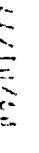




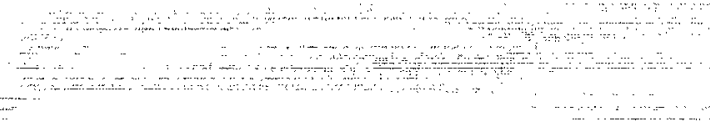

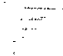

$(-2)$

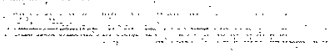

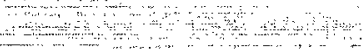

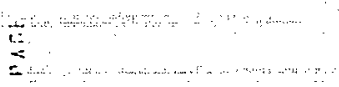

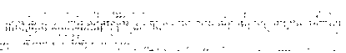

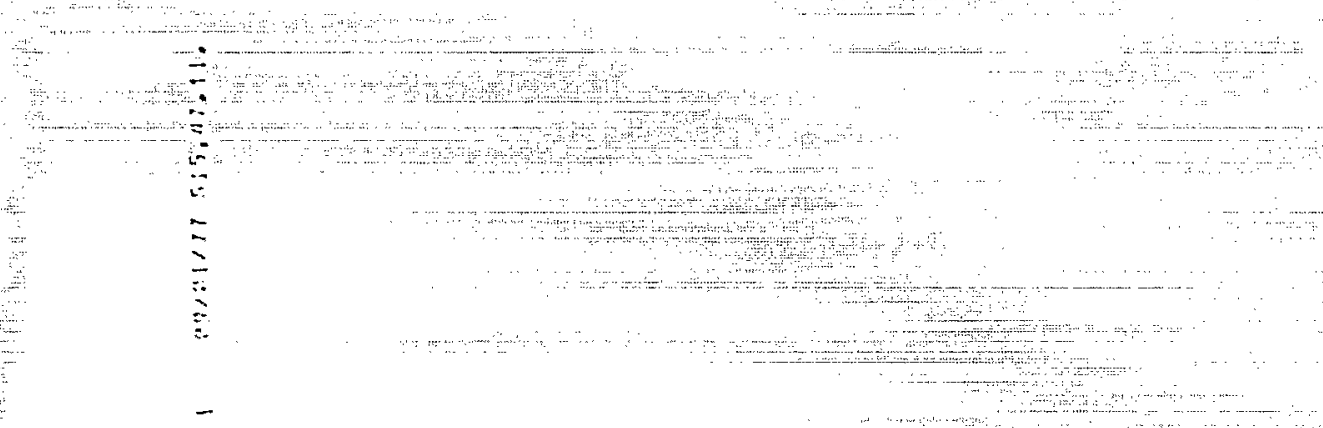

$-1+\cdots$

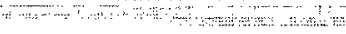

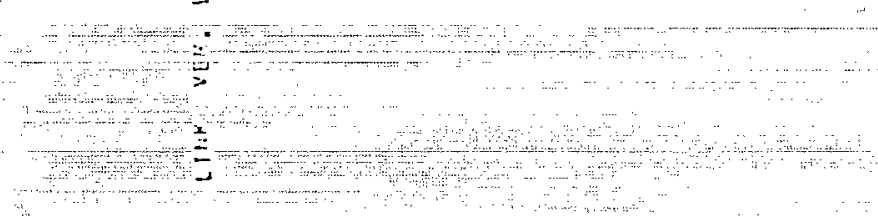

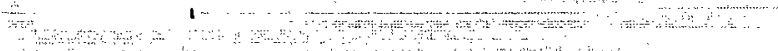

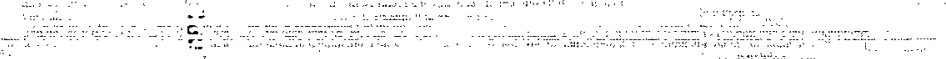

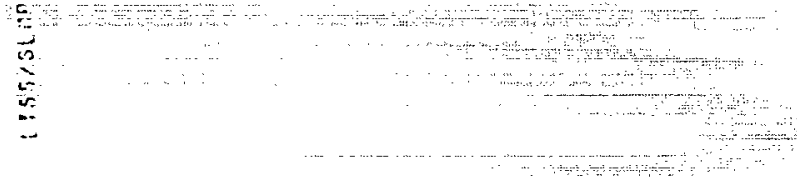

$+1$

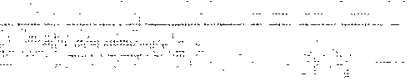

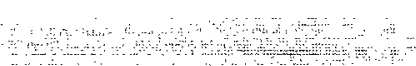

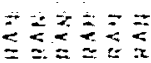

z

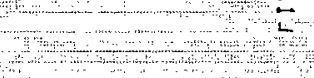

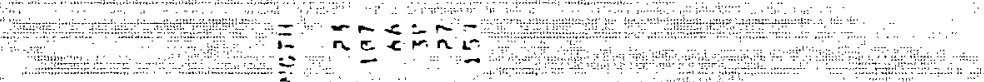

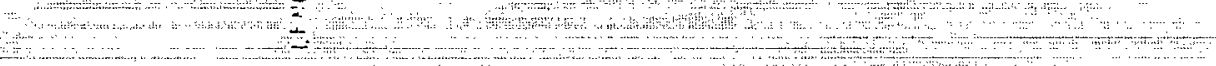

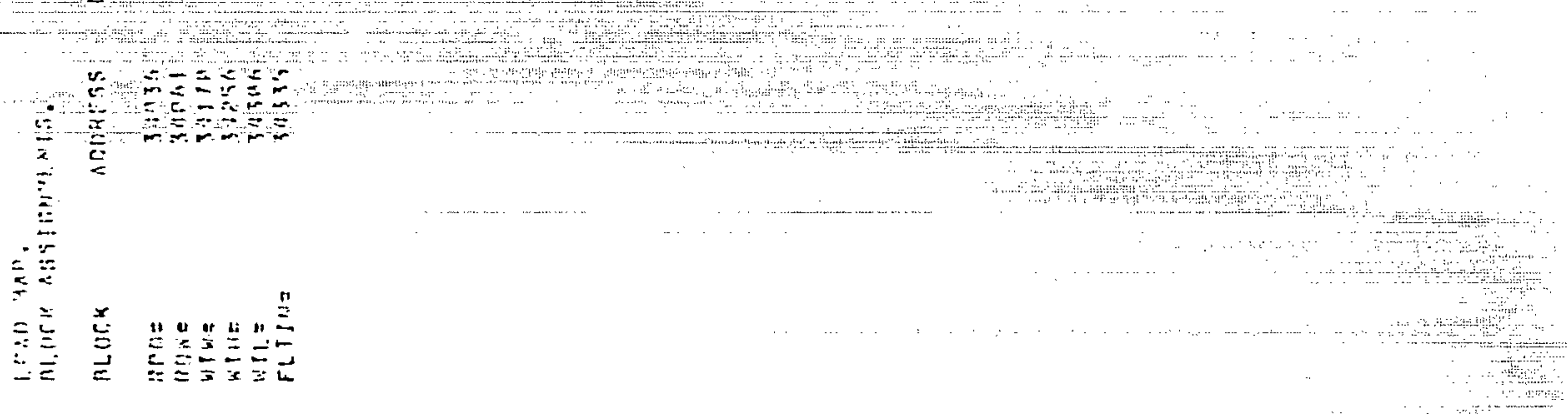

$=0$

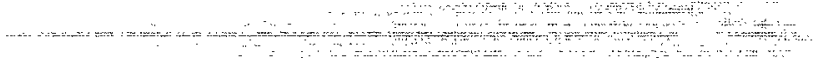

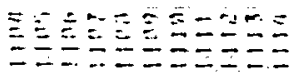

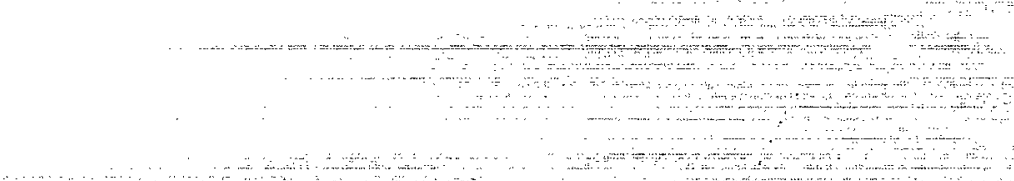


APPENDIX D

RICTL USER'S MANUAL

A sample FORTRAN Coding Form is attached showing data card entries. The title card (Card No. 1 ) is not included in this form,

If one desires to use English units, note that subroutine sTIF must express the coupler stiffnesses in English units. The English units for the coupler described by Fig. 2 are:

$$
\begin{aligned}
& \text { IF (U. } \left.{ }^{2 T}=8 .\right) \mathrm{SK}^{(\mathrm{NM})}=4000000 \\
& \text { IF }((U . \mathrm{LT},-5) \text { AND }(\mathrm{U}, \mathrm{GE}-\mathrm{-}-8 .)) \mathrm{SK}(\mathrm{NM})=120000 \text {. } \\
& \text { IF }((\mathrm{U}, \mathrm{LT}, 0 .) \text { AND }(\mathrm{U}, \mathrm{GE}-.5)) \mathrm{SK}(\mathrm{NM})=600000 \text {. } \\
& \text { IF ((U, LT. 1.). AND. (U, GE, 0.)) SK (NM) }=0.0 \\
& \text { IF ((U. LT.6.5). AND. (U. GE. 1.)) SK (NM) }=500000 \\
& \text { IF (U. GT. 6.5) SK (NM) }=700000 \text {. }
\end{aligned}
$$

The English units above are in inches for values of $U$, and $1 \mathrm{~b} / \mathrm{in}$. for values of SK (NM) .... The proper English units in the program are in, ib, and $\mathrm{s}$. The value of $g$ that must be input for English units is $386=\left(\mathrm{in} / \mathrm{s}^{2}\right), v_{\mathrm{I}}$ must be in in./s:

The metric units used in this report are $m, N$, and $s$. The value of $g$ input for metric units is $9.80665\left(\mathrm{~m} / \mathrm{s}^{2}\right) \quad V_{\mathrm{I}}$ must be in m/s.

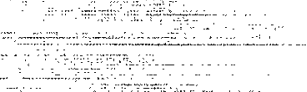

CARD INPUT

\section{Card 1 Title Card \\ Title Card is a descriptive title up to 80 characters.} Format ( $8 \mathrm{A10})$

Card 2 ICASE, VI, RF, NTS, DT, IOPT (1) -

ICASE $=1$, Car striking train free to rol1 format (I5)

$=2$, Car striking train with brakes Format (I5)

$=3$, Train striking car free to rol1 Format (I5)

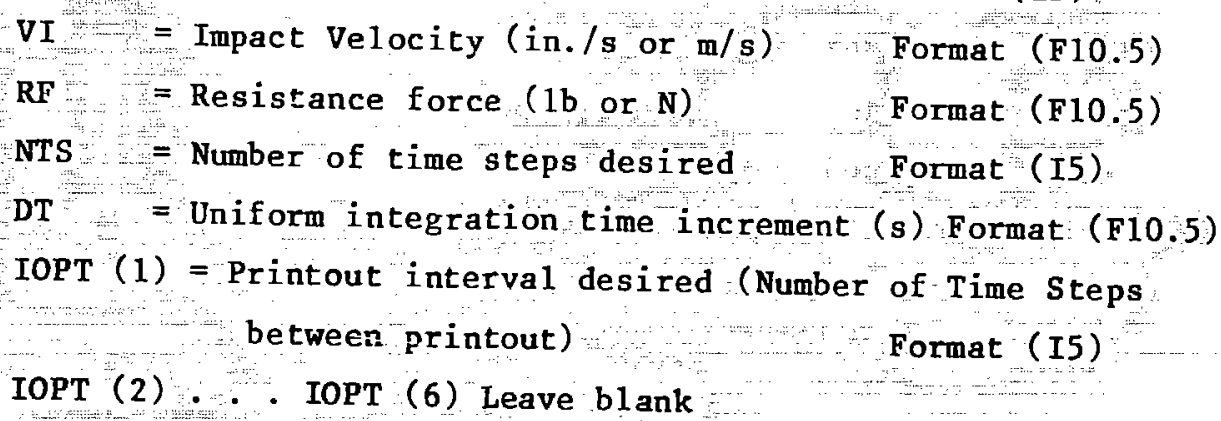


Cards $3,4 \times(I), I=1,2, \ldots ., 11$

$$
\begin{array}{r}
x(I)=\text { Longitudinal coordinate of mass lumps (in, m) } \\
\text { Format (F10.4) }
\end{array}
$$

Cards 5,6 y (I), I = 1, 2,... 11

$y(I)=$ Vertical coordinate of mass lumps (in, $m$ )

Format (F10.4)

Cards $7,8 \mathrm{w}(\mathrm{I}), \mathrm{I}=1,2, \ldots .11$

$\mathrm{w}(\mathrm{I})=$ Lumped weights $(1 \mathrm{~b}, \mathrm{~N}) \quad$ Format (E12.6)

Cards 9,10 I (?), $I=1,2, \ldots$. . 11*

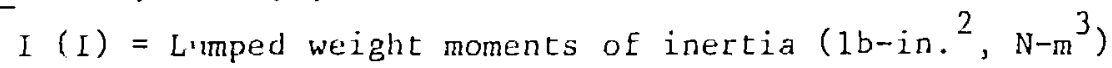

Firmat (E12.6)

* Note: I (LI) is not used and may be left blank.

Card 11 A12, A23, A34, A45, A56, A67, A89, A910

$A^{\prime} s=$ Cross-section areas of members (in. ${ }^{2}, m^{2}$ )

Format (F10.4)

Card 12 112, I23, I34, I45, I56, I67, I89, I910

$I^{\prime} s=$ Cross-section moments of inertia of members (in. ${ }^{4}, m^{4}$ )

Format (F10.4)

Card 13 Er, Ec, g

Er $\quad=$ Railcar Young's modulus of elasticity (1b/in. ${ }^{2}$, $\left.\mathrm{N} / \mathrm{m}^{2}\right) \quad$ Format (E12.6)

Ec = Container Young's modulus of elasticity (1b/in. ${ }^{2}$, $\mathrm{N} / \mathrm{m}^{2}$ ) Format (E12.6)

$\mathrm{g}=$ Acceleration due to gravity (386 in. $/ \mathrm{s}^{2}$, $9.80665 \mathrm{~m} / \mathrm{s}^{2}$ ) Format (E12.6)

Cards 14,15,16 Leave blank but include

Card $17 \quad \mathrm{C} 28, \mathrm{C} 38, \mathrm{C} 105, \mathrm{C} 106, \mathrm{C} 711$

$\mathrm{C}^{\prime} \mathrm{s}=$ Tiedown damping, $\mathrm{C} 711$ is coupler damping $\left(\frac{1 \mathrm{~b}-\mathrm{s}}{\mathrm{in} .}, \frac{\mathrm{N}-\mathrm{s}}{\mathrm{m}}\right)$

Format $(\mathrm{F} 10.4)$

Card $18 \quad \mathrm{~K} 28, \mathrm{~K} 38, \mathrm{~K} 105, \mathrm{~K} 106$

$\mathrm{K}^{\prime} \mathrm{s}=$ Tiedown axial stiffness (1b/in. N/m) Format (F10.4) 


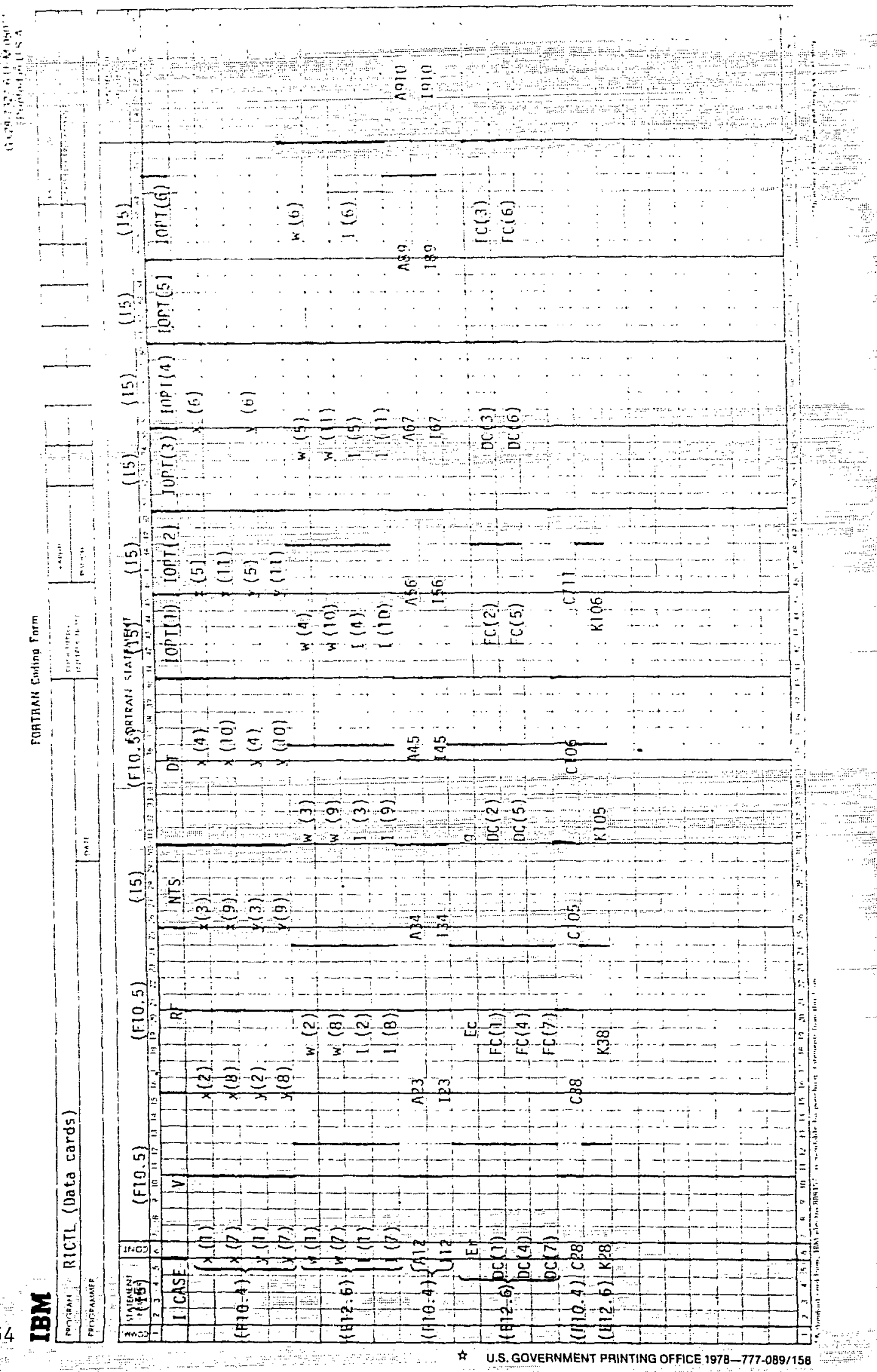

